\title{
Spin Hurwitz numbers and the Gromov-Witten invariants of Kähler surfaces
}

\author{
Junho Lee And Thomas H. Parker
}

\begin{abstract}
The classical Hurwitz numbers which count coverings of a complex curve have an analog when the curve is endowed with a theta characteristic. These "spin Hurwitz numbers," recently studied by Eskin, Okounkov and Pandharipande, are interesting in their own right. By the authors' previous work, they are also related to the Gromov-Witten invariants of Kähler surfaces. We prove a recursive formula for spin Hurwitz numbers, which then gives the dimension zero GW invariants of Kähler surfaces with positive geometric genus. The proof uses a degeneration of spin curves, an invariant defined by the spectral flow of certain anti-linear deformations of $\bar{\partial}$, and an interesting localization phenomenon for eigenfunctions that shows that maps with even ramification points cancel in pairs.
\end{abstract}

\section{Introduction}

The Hurwitz numbers of a complex curve $D$ count covers with specified ramification type. Specifically, consider degree $d$ (possibly disconnected) covering maps $f: C \rightarrow D$ with fixed ramification points $q^{1}, \ldots, q^{k} \in D$ and ramification given by $m^{1}, \ldots, m^{k}$ where each $m^{i}=\left(m_{1}^{i}, \ldots, m_{\ell_{i}}^{i}\right)$ is a partition of $d$. The Euler characteristic of $C$ is related to the genus $h$ of $D$ and the partition lengths $\ell\left(m^{i}\right)=\ell_{i}$ by the Riemann-Hurwitz formula

$$
\chi(C)=2 d(1-h)+\sum_{i=1}^{k}\left(\ell\left(m^{i}\right)-d\right) .
$$

In this context, there is an ordinary Hurwitz number

$$
\sum \frac{1}{|\operatorname{Aut}(f)|}
$$

that counts the covers $f$ satisfying (1.1) mod automorphisms; the sum depends only on $h$ and $\left\{m^{i}\right\}$. 
Now fix a theta characteristic $N$ on $D$, that is, a holomorphic line bundle with an isomorphism $N^{2}=K_{D}$ where $K_{D}$ is the canonical bundle of $D$. The pair $(D, N)$ is called a spin curve. By a well-known theorem of Mumford and Atiyah, the deformation class of the spin curve is completely characterized by the genus $h$ of $D$ and the parity

$$
p=(-1)^{h^{0}(D, N)}
$$

Now consider degree $d$ ramified covers $f: C \rightarrow D$ for which

- each partition $m^{i}$ is odd, i.e., each $m_{j}^{i}$ is an odd number.

In this case, the ramification divisor $\mathcal{R}_{f}$ of $f$ is even and the twisted pullback bundle

$$
N_{f}=f^{*} N \otimes \mathcal{O}\left(\frac{1}{2} \mathcal{R}_{f}\right)
$$

is a theta characteristic on $C$ with parity

$$
p(f)=(-1)^{h^{0}\left(C, N_{f}\right)}
$$

After choosing a spin curve $(D, N)$ and odd partitions $m^{1}, \ldots, m^{k}$, we can consider the total count of maps satisfying (1.1) modulo automorphisms, counting each map as \pm 1 according to its parity. This sum is also a deformation invariant of the spin curve $(D, N)$, so depends only on $h$ and $p$. Thus, we define the spin Hurwitz numbers of a spin curve $(D, N)$ of genus $h$ and parity $p$ to be

$$
H_{m^{1}, \ldots, m^{k}}^{h, p}=\sum \frac{p(f)}{|\operatorname{Aut}(f)|},
$$

where the sum is over all maps $f$, non-isomorphic as branched covers, satisfying (1.1).

Eskin et al. [3] gave a combinatorial method for finding the spin Hurwitz numbers when $D$ is an elliptic curve with the trivial theta characteristic (genus $h=1$ and parity $p=-1$ ). Our main result gives recursive formulas that express all other spin Hurwitz numbers (except the related $h=0$ and $h=p=1$ cases) in terms of the Eskin-Okounkov-Pandharipande numbers. The statement involves two numbers that are associated with partition $m=$ 
$\left(m_{1}, \ldots, m_{\ell}\right)$ of $d$, namely

$$
|m|=\prod m_{j} \quad \text { and } \quad m !=|\operatorname{Aut}(m)|,
$$

where $\operatorname{Aut}(m)$ is the subgroup of symmetric group $S_{\ell}$ permuting equal parts of the partition $m$ of $d$. We call a partition $m$ odd or even according to whether $|m|$ is odd or even.

Theorem 1.1. Fix $d>0$ and let $m^{1}, \ldots, m^{k}$ be a collection of odd partitions of $d$.

(a) If $h=h_{1}+h_{2}$ and $p \equiv p_{1}+p_{2}(\bmod 2)$ then for $0 \leq k_{0} \leq k$

$$
H_{m^{1}, \ldots, m^{k}}^{h, p}=\sum_{m}|m| m ! H_{m^{1}, \ldots, m^{k_{0}, m}}^{h_{1}, p_{1}} \cdot H_{m, m^{k_{0}+1}, \ldots, m^{k}}^{h_{2}, p_{2}}
$$

(b) If $h \geq 2$ or if $(h, p)=(1,+)$ then

$$
H_{m^{1}, \ldots, m^{k}}^{h, p}=\sum_{m}|m| m ! H_{m, m, m^{1}, \ldots, m^{k}}^{h-1, p}
$$

where the sums are over all odd partitions $m$ of $d$.

Gunningham [4] has used completely different methods to obtain results that overlap ours. He casts the spin Hurwitz numbers as a topological quantum field theory and obtains a formula for the spin Hurwitz numbers in terms of the coefficients of Sergeev algebras, which can be determined recursively. The exact relationship between Gunningham's result and ours - both the proof and the formulas - is not obvious and would be interesting to elucidate.

For us, spin Hurwitz numbers arose from studying the GW invariants of Kähler surfaces. The results of [10] shows that the GW invariants associated with dimension zero moduli spaces are exactly the etale spin Hurwitz numbers

$$
H_{d}^{h, p}=H_{\left(1^{d}\right)}^{h, p}
$$

that count degree $d$ etale covers, defined as above by taking $m$ to be the trivial partition $\left(1^{d}\right)$ of $d$. The precise relation can be described as follows.

Let $X$ be a Kähler surface with a smooth canonical divisor $D$. By the adjunction formula, the normal bundle $N \rightarrow D$ is a theta characteristic, so each component of $(D, N)$ is a spin curve. The results of $[8,10]$ show that the GW invariant of $X$ is a sum over the components of $(D, N)$ of certain local $G W$ invariants $G W_{g, n}^{\text {loc }}$. As usual, one can work either with the local 
GW invariants that count maps from connected domains of genus $g$ or with the local "Gromov-Taubes" invariants $G T_{g, n}^{\text {loc }}$ that count maps from possibly disconnected domains of Euler characteristic $\chi$. With the latter, the main formula of [10] reads

$$
G T_{\chi, n}(X, \beta)=\prod_{k}\left(i_{k}\right)_{*} G T_{\chi_{k}, n}^{\mathrm{loc}}\left(D_{k}, N_{k} ; d_{k}\right)
$$

where $i_{k}$ is the inclusion $D_{k} \subset X$.

Now, assume $(D, N)$ is a connected genus $h$ spin curve with parity $p$ and consider maps $f: C \rightarrow D$ where $\chi(C)=2 d(h-1)$. Then the space of degree $d$ stable maps with no marked points has dimension zero, both sides of (1.10) are rational numbers and, in fact, the dimension zero local GT local invariants are exactly the etale spin Hurwitz numbers:

$$
G T_{d}^{\mathrm{loc}, h, p}=H_{d}^{h, p}
$$

(the relation $\chi=2 d(h-1)$ is implicit in this notation). For $h=0,1$, these invariants were calculated for all degrees $d$ in $[8,10]$. As an immediate application of Theorem 1.1, one can express the local invariants (1.11) with $h \geq 2$ in terms of $h=1$ spin Hurwitz numbers calculated in [3]:

Theorem 1.2. Let $H_{m}$ denote the spin Hurwitz numbers $H_{m}^{1,-}$ where $m$ is one or more partitions. Then for $h \geq 2$ we have

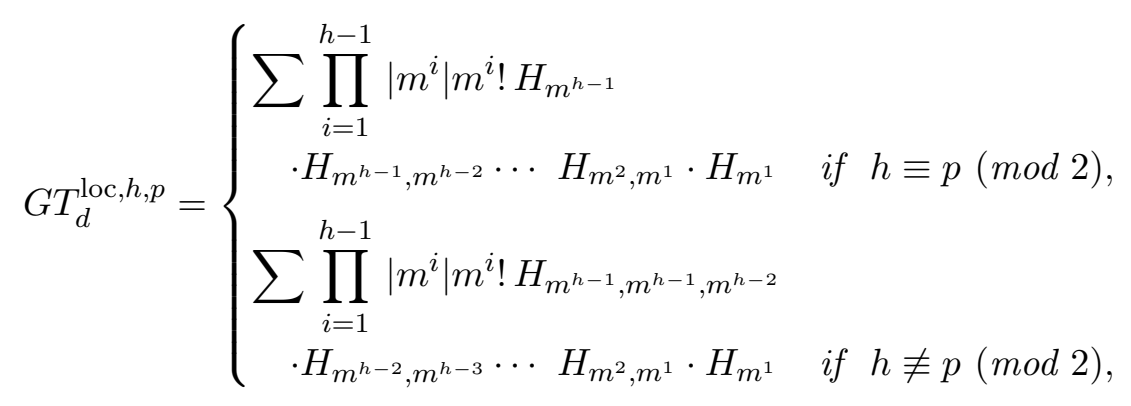

where the sums are over all odd partitions $m^{1}, \ldots, m^{h-1}$ of $d$.

The proof of Theorem 1.1 involves five main steps, described below. All are based on the observation that the $\bar{\partial}$-operators on spin bundles $N_{f}$ extend 
to a 1-parameter family of real operators

$$
L_{t}=\bar{\partial}+t R: \Omega^{0}\left(C, N_{f}\right) \rightarrow \Omega^{0,1}\left(C, N_{f}\right)
$$

with remarkable properties. The key idea is that the parity of a map $f$ is an isotopy invariant of the family $L_{t}$, and one can explicitly describe the behavior of the operators $L_{t}$ as both the domain and the target of $f$ degenerate to nodal spin curves. This allows us to express both the parity and the number of covering maps in terms of the maps into the irreducible components of the nodal target curve, giving the recursion formulas of Theorem 1.1.

STEP 1: Relating $L_{t}$ and parity. Section 2 gives a method for constructing complex anti-linear bundle maps $R$, which then define a family $L_{t}=\bar{\partial}+t R$ of operators as in (1.12). We then prove a vanishing theorem showing that ker $L_{t}=0$ for each stable map $f$ and each $t \neq 0$. This property was exploited in our previous work (e.g., [10], [11]) and underlies all later sections.

In Section 3, we express the parity as an isotopy invariant — the "TR spectral flow" - of the path of operators $L_{t}$. In this form, in contrast to the original definition (1.6), parity is unchanged under deformations. We also relate the parity to the determinant of $L_{t}$ on its low eigenspaces.

STEP 2: Degenerating spin curves and sum formulas. The Hurwitz numbers of $D$ can be viewed as the relative Gromov-Witten invariants of $D$ relative to a branch locus $\left\{q_{1}, \ldots, q_{\ell}\right\} \in D$. Under condition (1.1) the space of relative stable maps is a finite set corresponding to stable maps $f: C \rightarrow D$ branched over $\left\{q_{i}\right\}$. We then adopt the sum formula arguments of [7], as the first author has done in [9]. There are three parts of the argument:

- Identifying the maps $f: C \rightarrow D$ that occur as limits as $D$ degenerates to a nodal spin curve $D_{0}$.

- Constructing a family $\mathcal{C} \rightarrow \Delta$ of deformations of the maps $f: C_{0} \rightarrow D_{0}$.

- A gluing procedure that relates the moduli space of a general fiber to data along the central fiber.

In each step, it is necessary to keep track of the target curve, the domain curve, the map, the spin structures, and ultimately the spectral flow. The spin structure adds complication: in order to extend the spin structure across the central fiber it is necessary, following Cornalba [2], to insert a rational curve at each node as the target degenerates. Section 4 proves Theorem 1.1 assuming two deferred facts: the existence of a smooth family moduli space and a crucial statement (Theorem 4.2) about parities. 
STEP 3: Algebraic families of maps. The required family of maps is constructed in Section 5. The construction, which uses blowups and base changes, provides explicit coordinates for the analysis done in later sections. Extra steps are needed to ensure that there are line bundles on the family whose restrictions to the general fiber gives the spin structure $N$ on $D$ and (1.5) for each $f: C \rightarrow D$. Moreover, as shown in Section 6, there are antilinear bundle maps $R$, and hence operators $L_{t}=\bar{\partial}+t R$ on the family with the properties described in Section 2.

STEP 4: Eigenbundles of $L_{t}$ and parity for odd partitions. In Section 7, we switch from algebraic geometry to analysis and construct bundles of low eigenspaces of $L_{t}$. The formulas of Section 3 then apply on the family, giving a simple parity formula (Lemma 8.1) for odd partitions. But a complication arises for even partitions: the maps into $D_{0}$ may be ramified over the nodes in a way that does not satisfy (1.4), so the irreducible components of $D_{0}$ do not have well-defined spin Hurwitz numbers. Correspondingly, we obtain an analytic formula for the parity (Theorem 8.2) that must be evaluated at smooth curves.

STEP 5: Localization and cancellation. Finally, we exploit another remarkable property of the operators $L_{t}$ : as $t \rightarrow \infty$ there is a basis of the low eigenspace of $L_{t}^{*}$ consisting of "bump functions" sharply concentrated at the nodes, and $p(f)$ can be expressed in terms of $L^{2}$ inner products of these bump functions. The concentration allows us to pair up maps with even ramification and show that the contributions of the maps with even ramification cancel in pairs. This cancellation is the key observation of the paper and is the final ingredient in the proof of Theorem 1.1.

Section 11 presents some specific calculations: Theorem 1.1 is used to determine all spin Hurwitz numbers with degree $d=4$ for every genus.

To complete our program of calculating the GW invariants of Kähler surfaces with smooth canonical divisor, one needs to extend Theorem 1.2 to the cases with higher dimensional moduli spaces. As shown in [11], this requires calculating the Euler class of a real obstruction bundle arising from the pullback spin structure. We will apply the methods developed here to the higher-dimensional case in a future paper.

\section{Antilinear deformations of $\bar{\partial}$}

Let $f: C \rightarrow D$ be a holomorphic map of degree $d>0$ between smooth curves and let $N \rightarrow D$ be a theta characteristic. As shown in [10], there is a holomorphic 2-form on the total space of $N$ that induces a conjugate-linear 
bundle map $R: f^{*} N \rightarrow \bar{K}_{C} \otimes f^{*} N$ with several remarkable properties. In this section we use a different approach to produce a similar bundle map $R: N_{f} \rightarrow \bar{K}_{C} \otimes N_{f}$, where $N_{f}$ is the twisted pullback bundle (1.5). This map $R$ and the associated deformations $\bar{\partial}+t R$ of the $\bar{\partial}$-operator on $N_{f}$ are the central objects in this paper.

To start, let $D$ be a smooth curve with canonical bundle $K_{D}$ and a Riemannian metric. Let $N \rightarrow D$ be a holomorphic line bundle with a Hermitian metric $\langle$,$\rangle , conjugate linear in the second factor. Let ()=,\operatorname{Re}\langle$,$\rangle be$ the corresponding positive definite inner product, and let $\bar{*}: \Lambda^{p, q} D \otimes N^{*} \rightarrow$ $\Lambda^{1-p, 1-q} D \otimes N$ be the associated conjugate-linear star operator.

Lemma 2.1. Any holomorphic section $\varphi$ of $K_{D} \otimes\left(N^{*}\right)^{2}$ induces a bundle map

$$
R: N \rightarrow \bar{K}_{D} \otimes N
$$

that, with its adjoint $R^{*}$ with respect to the inner product (, ), satisfies

$$
\text { (a) } R J=-J R
$$

(b) $R^{*} R=|\varphi|^{2} I d$

(c) $\bar{\partial}^{*} R+R^{*} \bar{\partial}=0$.

Proof. Regard $\varphi$ as a complex bundle map $\varphi: N \rightarrow K_{D} \otimes N^{*}$ and set $R=$ $\bar{*} \circ \varphi$. Because $\bar{*}$ is conjugate-linear, this immediately gives $R J=-J R$. Fix a point $p$, a local holomorphic coordinate $z$ around $p$ in which the metric is Euclidean to second order, and a local holomorphic section $\nu$ of $N$ with $|\nu(p)|=1$. It suffices to verify that (b) and (c) hold at $p$.

Let $\nu^{*}$ denote the dual section to $\nu$ and write $\varphi(\nu)=g d z \nu^{*}$. Then $\bar{\partial} g=0$ because $\varphi$ is holomorphic, and at $p$ we have $R(\nu)=\bar{g} d \bar{z} \nu$ and, taking the adjoint using the real inner product, $R^{*}(d \bar{z} \nu)=\bar{g} \nu$. It follows that $R^{*} R=$ $|g|^{2} I d=|\varphi|^{2} I d$. Choosing an arbitrary section $\xi=f \nu$ of $N$, we have

$$
R^{*} \bar{\partial} \xi=R^{*}\left(\frac{\partial f}{\partial \bar{z}} d \bar{z} \nu\right)=\overline{\left(\frac{\partial f}{\partial \bar{z}}\right)} R^{*}(d \bar{z} \nu)=\bar{g} \frac{\partial \bar{f}}{\partial z} \nu
$$

at $p$. On the other hand, the formulas $\bar{\partial}^{*}=-\bar{*} \bar{\partial} \bar{*}$ and $(\bar{*})^{2}=-1$ on $\Omega^{0,1}\left(N^{*}\right)$ show that at $p$

$$
\bar{\partial}^{*} R \xi=-\bar{*} \bar{\partial} \bar{*} \bar{*} \varphi(f \nu)=\bar{*} \bar{\partial}\left(f g d z \nu^{*}\right)=\bar{g} \frac{\partial \bar{f}}{\partial z} \bar{*}\left(d \bar{z} d z \nu^{*}\right)=-\bar{g} \frac{\partial \bar{f}}{\partial z} \nu .
$$

This cancels (2.3), giving statement (c).

An endomorphism $R$ as in (2.1) determines a 1-parameter family of perturbations of the $\bar{\partial}$-operator, namely the operators $L_{t}: \Omega^{0}(N) \rightarrow \Omega^{0,1}(N)$ 
defined by

$$
L_{t}=\bar{\partial}+t R \quad t \in \mathbb{R}
$$

Properties (2.2) imply a remarkably simple vanishing theorem.

Vanishing Theorem 2.2. If $R$ satisfies (2.2) with $\varphi \not \equiv 0$, then $\operatorname{ker} L_{t}=0$ for each $t \neq 0$.

Proof. If $L_{t} \psi=0$ then by $(2.2)$ we have

$$
0=\int_{D}\left|L_{t} \xi\right|^{2}=\int_{D}|\bar{\partial} \xi|^{2}+|R|^{2}|\xi|^{2} .
$$

Thus $\xi$ is holomorphic and vanishes on the open set where $R=\varphi \neq 0$, so $\xi \equiv 0$.

Many of the results in subsequent sections can be viewed as natural extensions of Theorem 2.2. For a first extension, let $f: C \rightarrow D$ be a holomorphic map with ramification points $q_{j}$ and ramification divisor $\mathcal{R}_{f}=$ $\sum\left(m_{j}-1\right) q_{j}$. If $N$ is a theta characteristic on $D$ and $A$ is any divisor on $C$, we can consider the twisted bundle

$$
N_{f}=f^{*} N \otimes \mathcal{O}_{C}(A)
$$

on $C$. We then have the following relative version of Lemma 2.1.

Corollary 2.3. A holomorphic section $\varphi$ of $\mathcal{O}\left(\mathcal{R}_{f}-2 A\right)$ induces a bundle map

$$
R_{f}: N_{f} \rightarrow \bar{K}_{C} \otimes N_{f}
$$

that satisfies properties (2.2), and the Vanishing Theorem 2.2 applies to $L_{t}=\bar{\partial}+t R_{f}$.

Proof. The Hurwitz formula and the isomorphism $N^{2}=K_{D}$ induce an isomorphism $K_{C} \otimes\left(N_{f}^{*}\right)^{2}=\mathcal{O}\left(\mathcal{R}_{f}-2 A\right)$, so we can apply Lemma 2.1.

\section{Parity as the TR spectral flow}

Suppose that $A_{t}: V_{t} \rightarrow W_{t}$ is a smooth path of linear maps where $V_{t}$ and $W_{t}$ are the fibers of real vector bundles $V$ and $W$ over $\mathbb{R}$. The real variety 
$\mathcal{S} \subset \operatorname{Hom}(V, W)$ of non-invertible maps separates the bundle $\operatorname{Hom}(V, W)$ into connected open sets called chambers. If $A_{t_{1}}$ and $A_{t_{2}}$ are non-singular, the mod 2 spectral flow of the path $A_{t}$ from $t_{1}$ to $t_{2}$ is calculated by perturbing the family to be transversal to $\mathcal{S}$ and counting the number of times the family crosses $\mathcal{S}$ modulo 2 ; this is independent of the perturbation. This section describes a modified spectral flow that applies to the operators $L_{t}=\bar{\partial}+t R$ of (2.6).

We begin with a definition that occurs in quantum mechanics. Let $V$ and $W$ be real vector bundles over $\mathbb{R}$. A $T R$ ("time-reversal") structure is a lift of the map $t \mapsto-t$ to bundle maps $T: V \rightarrow V$ and $T: W \rightarrow W$ satisfying $T^{2}=-I d$. A bundle map $A: V \rightarrow W$ is $T R$ invariant if there is a $T$ as above such that

$$
[A, T]=0 \text { that is, } A_{-t}=T_{t} A_{t} T_{t}^{-1} .
$$

In particular, $T_{0}=J$ is a complex structure on $V_{0}$ and $W_{0}$ and by (3.1) and $A_{0}$ is complex linear.

Let $\mathcal{T} R$ denote the space of all smooth TR invariant $A: V \rightarrow W$ that are invertible except at finitely many values of $t$. For an open dense set of $A \in \mathcal{T} R, A_{0}$ is non-singular and $A$ intersects $\mathcal{S}$ transversally at finitely many points $\left\{ \pm t_{i}\right\}$ (proof: given $A$, perturb $A_{t}$ for $t \geq 0$ to $A_{t}^{\prime}$ transverse to $\mathcal{S}$ and with $A_{0}^{\prime}$ complex and invertible, then define $A_{-t}^{\prime}$ by (3.1) and smooth, symmetrically in $t$, around $t=0$ ). Thus the mod 2 spectral flow from $t=-\infty$ to $t=\infty$ is well-defined, but is 0 because the singular points are symmetric. However, there is a well-defined TR spectral flow

$$
S F^{T R}: \mathcal{T} R \rightarrow\{ \pm 1\}
$$

defined for $A \in \mathcal{T} R$ by perturbing to a generic $C \in \mathcal{T} R$ and setting $S F^{T R}(A)=(-1)^{s}$ where $s$ is the mod 2 spectral flow of $C$ from $t=0$ to $t=\infty$. Regarding $C$ as a path in $\operatorname{Hom}(V, W), s$ is the mod 2 intersection $C \cap \mathcal{S}$, which depends only on the homology class is $C$. If $D$ is another generic perturbation with $s^{\prime}=D \cap \mathcal{S}$, then $s-s^{\prime}=\gamma \cap \mathcal{S}$ where $\gamma$ is a path from $B_{0}$ to $C_{0}$. But then $s-s^{\prime}$ is even because $B_{0}$ to $C_{0}$ are complex linear isomorphisms. Thus $S F^{T R}(A)$ is independent of the perturbation. In practice, two formulas are useful:

(i) If $V$ and $W$ both have finite rank $r$, the complex orientation on $V_{0}$ and $W_{0}$ extends to orient all fibers of $V$ and $W$. This means that $\operatorname{sgn} \operatorname{det} A_{t}$ is canonically defined for every $A \in \mathcal{T} R$ and all $t$. For generic $A \in \mathcal{T} R$ the sign of $\operatorname{det} A_{t}$ is positive for $t=0$ and changes sign with each 
transversal crossing of $\mathcal{S}$. Thus

$$
S F^{T R}(A)=\operatorname{sgn} \operatorname{det} A_{t}
$$

whenever $A_{s}$ is non-singular for all $s \geq t$.

(ii) Now suppose that $\operatorname{ker} A_{0}$ is finite-dimensional and $\dot{A}_{0}$ restricts to an isomorphism $B: \operatorname{ker} A_{0} \rightarrow$ coker $A_{0}$. Then ker $A_{0}$ and coker $A_{0}$ are complex vector spaces of the same dimension $d$. Choose a complexlinear map $C: \operatorname{ker} A_{0} \rightarrow$ coker $A_{0}$ and perturb $A$ to a generic $A^{\prime} \in \mathcal{T} R$ as above with $A_{0}^{\prime}=A_{0}+\varepsilon C$ and $A^{\prime}(t)=A_{t}$ for all $t \geq \delta$. Then $\operatorname{det} C>$ 0 and the mod 2 spectral flow of $A_{t}^{\prime}$ from $t=0$ to $t=\delta$ is sgn $\operatorname{det} B$. But one sees by differentiating (3.1) that $B$ satisfies $J B=-B J$; therefore $\operatorname{det} B=(-1)^{d}$ because the eigenvalues of $B$ come in pairs $\pm \sqrt{-1} \lambda_{i}$. We conclude that

$$
S F^{T R}(A)=(-1)^{\operatorname{dim}_{\mathbb{C}} \operatorname{ker} A_{0}} .
$$

The TR spectral flow readily applies to the operators introduced in Section 3. Let $(D, N)$ be a smooth spin curve with a bundle map $R$ as in Lemma 2.1 that is non-zero almost everywhere. For each $t, L_{t}=\bar{\partial}+t R$ extends to a Fredholm map

$$
L_{t}: V_{\mathbb{C}} \rightarrow W_{\mathbb{C}}
$$

from the Sobolev $W^{1,2}$ completion of $\Omega^{0}(N)$ to the $L^{2}$ completion of $\Omega^{0,1}(N)$. By elliptic theory, $V_{\mathbb{C}}$ (resp. $W_{\mathbb{C}}$ ) decomposes into finite-dimensional real eigenspaces $E_{\lambda}$ of $L_{t}^{*} L_{t}$ (resp. $\left.L_{t} L_{t}^{*}\right)$ whose eigenvalues $\{\lambda\}$ are real, nonnegative, discrete, and vary continuously with $t$. For each $t$, let $V_{t} \subset V_{\mathbb{C}}$ and $W_{t} \subset W_{\mathbb{C}}$ be the closure of the real span of the eigenspaces; these form vector bundles $V, W$ over $\mathbb{R}$. By Property (2.2a) we have

$$
J L_{t} J^{-1}=-J(\bar{\partial}+t R) J=-J(J \bar{\partial}-t J R)=L_{-t} .
$$

Thus, $T=J$ is a TR structure and $L=\left\{L_{t}\right\}$ is a TR-invariant operator.

To calculate the invariant (3.2), we can reduce to a finite-dimensional situation. Fix $\lambda_{0}>0$ not in the spectrum of $\bar{\partial}^{*} \bar{\partial}$ and define the low eigenspaces of $L_{t}^{*} L_{t}$ and $L_{t} L_{t}^{*}$ by setting

$$
E_{t}=\bigoplus_{\lambda<\lambda_{0}} E_{\lambda} \quad \text { and } \quad F_{t}=\bigoplus_{\lambda<\lambda_{0}} F_{\lambda} .
$$


These form finite-rank real vector bundles $E \subset V$ and $F \subset W$ over an interval $[-\delta, \delta]$ where $\lambda_{0}$ remains outside the spectrum, and (3.5) again shows that $L: E \rightarrow F$ is TR-invariant.

Theorem 3.1. The parity of a spin structure $(D, N)$ is the TR spectral flow of the Fredholm operator $L: V \rightarrow W$, and for $0<|t|<\delta$ it is also the determinant of the finite-dimensional operator $L_{t}: E_{t} \rightarrow F_{t}$ :

$$
p=S F^{T R}(L) \quad \text { and } \quad S F^{T R}(L)=\operatorname{sgn} \operatorname{det} L_{t} \quad \text { for }|t| \leq \delta .
$$

Proof. By its definition (1.6), the parity is $p=(-1)^{h}$ where $h=\operatorname{dim}_{\mathbb{C}} \operatorname{ker} \bar{\partial}=$ $\operatorname{dim}_{\mathbb{C}} \operatorname{ker} L_{0}$. Observe that $\dot{L}_{0}=R$ is injective on ker $\bar{\partial}$ by Theorem 2.2 , and hence is an isomorphism because Riemann-Roch shows that dimker $\bar{\partial}-$ dim coker $\bar{\partial}=\chi(D, N)=0$. The first equality therefore follows by (3.4). For all $-\delta \leq t \leq \delta, L_{t}^{*} L_{t}$ is non-singular on the eigenspaces with $\lambda>\lambda_{0}$, so the spectral flow is determined by the restriction of $L_{t}$ to the low eigenspaces (3.6), where it is given by formula (3.3).

As a corollary, we obtain a simple proof of the Atiyah-Mumford Theorem on spin structures.

Corollary 3.2. Parity is an isotopy invariant of spin structures $(D, N)$.

Proof. If $\left(D_{s}, N_{s}\right)$ is a path of spin curves then $K_{D_{s}}\left(N_{s}^{*}\right)^{2}=\mathcal{O}$ is trivial for each $s$, so there are smoothly varying nowhere-zero maps $R_{s}$ as in Lemma 2.1. For fixed $t \neq 0$, Theorem 2.2 shows that $L_{s}=\bar{\partial}+t R_{s}$ is injective for all $s$, so $S F^{T R}\left(L_{s}\right)$ - and hence the parity — is independent of $s$.

In Sections 5 to 8 we will extend this proof by incorporating maps as in Corollary 2.3 and applying it to families of spin curves that degenerate to nodal curves.

\section{Degeneration, gluing and the proof of Theorem 1.1}

The proof of Theorem 1.1 is based on the method of [9]: we express the spin Hurwitz numbers in terms of relative Gromov-Witten moduli space and apply the limiting and gluing arguments of [7] for a degeneration of spin curves to form a family of moduli spaces. We then use a smooth model of the family of moduli spaces to calculate parities. The calculation immediately yields the desired recursion formula. This section outlines the proof, drawing on two facts that are deferred: the construction of a smooth model (done in Sections 5 and 6), and the computation of parities (done in Sections 8-10). 
As in [9], we begin by expressing the spin Hurwitz numbers (1.7) in terms of GW relative moduli spaces (cf. [6]). Let $D$ be a smooth curve of genus $h$ and let $V=\left\{q^{1}, \ldots, q^{k}\right\}$ be a fixed set of points on $D$. Given partitions $m^{1}, \ldots, m^{k}$ of $d$, a degree $d$ holomorphic map $f: C \rightarrow D$ from a (possibly disconnected) curve $C$ is called $V$-regular with contact partitions $m^{1}, \ldots, m^{k}$ if, for each $i=1, \ldots, k, f^{-1}\left(q^{i}\right)$ consists of $\ell\left(m^{i}\right)$ points $q_{j}^{i}$ so that the ramification index of $f$ at $q_{j}^{i}$ is $m_{j}^{i}$. If $m_{j}^{i}>1$ then the contact marked point $q_{j}^{i}$ is a ramification point of $f$ and $q^{i}$ is a branch point. The relative moduli space

$$
\mathcal{M}_{\chi, m^{1}, \ldots, m^{k}}^{V}(D, d)
$$

consists of isomorphism classes of $V$-regular maps $\left(f, C ;\left\{q_{j}^{i}\right\}\right)$ with contact vectors $m^{1}, \ldots, m^{k}$. Here $\chi(C)=\chi$ and all marked points are contact marked points. Since no confusion can arise, we will often write $\left(f, C ;\left\{q_{j}^{i}\right\}\right)$ simply as $f$.

Spin Hurwitz numbers are associated with those moduli spaces (4.1) that have (formal) dimension 0 . Thus we will henceforth assume that

$$
\operatorname{dim}_{\mathbb{C}} \mathcal{M}_{\chi, m^{1}, \ldots, m^{k}}^{V}(D, d)=2 d(1-h)-\chi-\sum_{i=1}^{k}\left(d-\ell\left(m^{i}\right)\right)=0
$$

With this assumption, all ramification points of a $V$-regular map $\left(f, C ;\left\{q_{j}^{i}\right\}\right)$ in (4.1) are contact marked points. In this case, forgetting the contact marked points gives a (ramified) cover $f$ satisfying (1.1). If $m^{i}=\left(1^{d}\right)$ for some $1 \leq i \leq k$ then

$$
H_{m^{1}, \ldots, m^{k}}^{h, p}=\frac{1}{\prod m^{i} !} \sum p(f)
$$

the sum is over all $f$ in (4.1) and $p(f)$ is the associated parity (1.6) (cf. Lemma 1.1 of [9]).

Adding trivial partitions does not change the formulas (1.1) and (4.2). It also does not change the spin Hurwitz numbers, namely,

$$
H_{\left(1^{d}\right), m^{1}, \ldots, m^{k}}^{h, p}=H_{m^{1}, \ldots, m^{k}}^{h, p} .
$$

Below, we fix $h, d, \chi$ and odd partitions $m^{1}, \ldots, m^{k}$ of $d$ so that the dimension formula (4.2) holds. In light of (4.3), we will add trivial partitions $m^{k+1}=m^{k+2}=m^{k+3}=\left(1^{d}\right)$ to make our discussion simpler. 
To adapt the main argument of [7] we will build a degeneration of target curves. Let $D_{0}=D_{1} \cup E \cup D_{2}$ be a connected nodal curve of arithmetic genus $h$ of a rational curve $E$ and smooth curves $D_{1}$ and $D_{2}$ of genus $h_{1}$ and $h_{2}$ with $h_{1}+h_{2}=h$, joined at nodes $p^{1}=D_{1} \cap E$ and $p^{2}=D_{2} \cap E$. Fix $k+3$ points $q^{i}$, all distinct and distinct from $p^{1}$ and $p^{2}$, with

$$
q^{k+1}, q^{1}, \ldots, q^{k_{0}} \in D_{1}, \quad q^{k+2} \in E, \quad q^{k_{0}+1}, \ldots, q^{k}, q^{k+3} \in D_{2} .
$$

where $0 \leq k_{0} \leq k$. In Section 5 , we will construct a deformation of $D_{0}$ with sections: a smooth complex surface $\mathcal{D}$ fibered over the disk $\Delta$ with parameter $r$ so that the central fiber is $D_{0}$ and the fibers $D_{r}$ with $r \neq 0$ are smooth curves of genus $h$ and $Q^{i}(0)=q^{i}$ for $1 \leq i \leq k+3$.

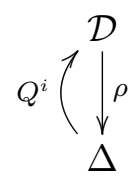

For each partition $m$ of $d$, consider the moduli space of maps

$$
\begin{aligned}
\mathcal{P}_{m}= & \mathcal{M}_{\chi_{1}, m^{k+1}, m^{1}, \ldots, m^{k_{0}, m}}^{V_{1}}\left(D_{1}, d\right) \times \mathcal{M}_{\chi_{e}, m, m^{k+2}, m}^{V_{e}}(E, d) \\
& \times \mathcal{M}_{\chi_{2}, m, m^{k_{0}+1}, \ldots, m^{k}, m^{k+3}}^{V_{2}}\left(D_{2}, d\right)
\end{aligned}
$$

where $V_{1}=\left\{q^{k+1}, q^{1}, \ldots, q^{k_{0}}, p^{1}\right\}, V_{e}=\left\{p^{1}, q^{k+2}, p^{2}\right\}, V_{2}=\left\{p^{2}, q^{k_{0}+1}, \ldots\right.$, $\left.q^{k}, q^{k+2}\right\}$ and

$$
\chi_{1}+\chi_{e}+\chi_{2}-4 \ell(m)=\chi
$$

For simplicity, let $\mathcal{M}_{m}^{1}, \mathcal{M}_{m}^{e}$ and $\mathcal{M}_{m}^{2}$ denote the first, second and third factors of $\mathcal{P}_{m}$. By (4.7) and our assumption that the dimension formula (4.2) holds, it is easy to see that whenever the space $\mathcal{P}_{m}$ is not empty, the relative moduli spaces $\mathcal{M}_{m}^{1}, \mathcal{M}_{m}^{e}$ and $\mathcal{M}_{m}^{2}$ all have dimension zero. In particular, $\chi_{e}=2 \ell(m)$ and

$$
\left|\mathcal{M}_{m}^{e}\right|=\frac{d ! m !}{|m|}
$$

where $\left|\mathcal{M}_{m}^{e}\right|$ denotes the cardinality of $\mathcal{M}_{m}^{e}$ (cf. Section 2 of [9]).

For $\left(f_{1}, f_{e}, f_{2}\right) \in \mathcal{P}_{m}$, let $x_{j}^{i}$ and $y_{j}^{i}$ be contact marked points of $f_{i}$ and $f_{e}$ over $p^{i} \in D_{i} \cap E$ with multiplicity $m_{j}$ where $i=1,2$ and $j=1, \ldots, \ell(m)$. By identifying $x_{j}^{i}$ with $y_{j}^{i}$, one can glue the domains of $f_{i}$ and $f_{e}$ to obtain a 
map $f: C \rightarrow D_{0}$ with $\chi(C)=\chi$. For notational convenience, we will often write the glued map $f$ as $f=\left(f_{1}, f_{e}, f_{2}\right)$. Denote by

$$
\mathcal{M}_{m, 0}
$$

the space of such maps $f=\left(f_{1}, f_{e}, f_{2}\right)$. Identifying contact marked points associates to each node of $C$ a multiplicity $m_{j}$ labeled by $j$. But the nodes of $C$ are not labeled. One can thus see that gluing domains gives a degree $(m !)^{2}$ covering map:

$$
\mathcal{P}_{m} \rightarrow \mathcal{M}_{m, 0}
$$

Remark 4.1. Let $f=\left(f_{1}, f_{e}, f_{2}\right)$ be a map in $\mathcal{M}_{m, 0}$. For $i=1,2$, since $\mathcal{M}_{m}^{i}$ has dimension zero, (i) the ramification points of $f_{i}$ are either contact marked points or nodal points of the domain of $f$, (ii) $f_{i}$ can have even ramification points only at nodal points and (iii) the number of even ramification points of $f_{i}$ is even.

For $r \neq 0$, consider the moduli spaces of $V$-regular maps into $D_{r}$, which we denote by

$$
\mathcal{M}_{r}=\mathcal{M}_{\chi, m^{1}, \ldots, m^{k+3}}^{V_{r}}\left(D_{r}, d\right) \quad \text { where } V_{r}=\left\{Q^{1}(r), \ldots, Q^{k+3}(r)\right\}
$$

By Gromov convergence, a sequence of holomorphic maps into $D_{r}$ with $r \rightarrow 0$ limits has a subsequence that converges to a map into $D_{0}$. Denote the set of such limits by

$$
\lim _{r \rightarrow 0} \mathcal{M}_{r}
$$

Lemma 3.1 of [9] shows that

$$
\lim _{r \rightarrow 0} \mathcal{M}_{r} \subset \bigcup_{m} \mathcal{M}_{m, 0}
$$

where the union is over all partitions $m$ of $d$ with $\mathcal{P}_{m} \neq \emptyset$.

Conversely, by the Gluing Theorem of [7], the domain of each map in $\mathcal{M}_{m, 0}$ can be smoothed to produce maps in $\mathcal{M}_{r}$ for small $|r|$. Shrinking $\Delta$ if necessary, for $r \in \Delta$, one can assign to each $f_{r} \in \mathcal{M}_{r}$ a partition $m$ of $d$ by (4.12). Let $\mathcal{M}_{m, r}$ be the set of all pairs $\left(f_{r}, m\right)$ and for each $f \in \mathcal{M}_{m, 0}$ 
denote by

$$
\mathcal{Z}_{m, f} \rightarrow \Delta
$$

the connected component of $\bigcup_{r \in \Delta} \mathcal{M}_{m, r} \rightarrow \Delta$ that contains $f$. It follows that

$$
\mathcal{M}_{r}=\bigsqcup_{m} \bigsqcup_{f_{r} \in \mathcal{M}_{m, 0}} \mathcal{Z}_{m, f, r} \quad(r \neq 0),
$$

where $\mathcal{Z}_{m, f, r}$ is the fiber of (4.13) over $r \in \Delta$. The Gluing Theorem shows that one can smooth each node $x_{j}^{i}=y_{j}^{i}$ of the domain of $f$, where $i=1,2$ and $j=1, \ldots, \ell(m)$, in $m_{j}$ ways to produce $|m|^{2}$ maps in $\mathcal{M}_{m, r}$, (there are two nodes on the target, the fiber $\mathcal{Z}_{m, f, r}(r \neq 0)$ consists of $|m|^{2}$ maps).

We now introduce a spin structure on $\rho: \mathcal{D} \rightarrow \Delta$ assuming that $\mathcal{D}$ is smooth. Given parities $p, p_{1}$ and $p_{2}$ with $p_{1}+p_{2}=p(\bmod 2)$, Cornalba's [2] constructs a line bundle $\mathcal{L} \rightarrow \mathcal{D}$ and a homomorphism

$$
\Phi: \mathcal{L}^{2} \rightarrow K_{\mathcal{D}}
$$

whose restrictions satisfy the following properties:

(a) For $r \neq 0, \mathcal{L}$ restricts to a theta characteristic on $D_{r}$ with a parity $p$ and $\Phi$ restricts to an isomorphism $\left(\left.\mathcal{L}\right|_{D_{r}}\right)^{2} \rightarrow K_{D_{r}}$.

(b) $\Phi$ vanishes identically on $E$ and $\left.\mathcal{L}\right|_{E}=\mathcal{O}_{E}(1)$.

(c) For $i=1,2, \mathcal{L}$ restricts to a theta characteristic on $D_{i}$ with parity $p_{i}$, and $\Phi$ restricts to an isomorphism $\left(\left.\mathcal{L}\right|_{D_{i}}\right)^{2} \rightarrow K_{D_{i}}$.

The pair $(\mathcal{L}, \Phi)$ is called a spin structure on $\rho: \mathcal{D} \rightarrow \Delta$.

Let $f=\left(f_{1}, f_{e}, f_{2}\right)$ be a map in $\mathcal{M}_{m, 0}$. Note that all ramification points of maps in $\mathcal{Z}_{m, f, r}(r \neq 0)$ have odd ramification indices since $m^{1}, \ldots, m^{k}$ are odd partitions. So, each map $f_{r}$ in $\mathcal{Z}_{m, f, r}$ has an associated parity $p\left(f_{r}\right)$ defined as in (1.6) by the pullback bundle $f_{r}^{*}\left(\left.\mathcal{L}\right|_{D_{r}}\right)$ and its ramification divisor $\mathcal{R}_{f_{r}}$. When the partition $m$ is odd, $f_{i}(i=1,2)$ also have associated parities $p\left(f_{i}\right)$ defined by $f_{i}^{*}\left(\left.\mathcal{L}\right|_{D_{i}}\right)$ and $\mathcal{R}_{f_{i}}$. In this context, (4.3), (4.4) and (4.14) shows that for $r \neq 0$ we have

$$
\begin{aligned}
H_{m^{1}, \ldots, m^{k}}^{h, p} & =H_{m^{1}, \ldots, m^{k},\left(1^{d}\right),\left(1^{d}\right),\left(1^{d}\right)}^{h, p} \\
& =\frac{1}{(d !)^{3} \prod_{i=1}^{k} m^{i} !} \sum_{m} \sum_{f \in \mathcal{M}_{m, 0}} \sum_{f_{r} \in \mathcal{Z}_{m, f, r}} p\left(f_{r}\right) .
\end{aligned}
$$


In Sections 5 to 10, we will establish the following facts about the parity.

Theorem 4.2. Let $f=\left(f_{1}, f_{e}, f_{2}\right) \in \mathcal{M}_{m, 0}$ and $r \neq 0$.

(a) If $m$ is odd, then $p\left(f_{r}\right)=p\left(f_{1}\right) p\left(f_{2}\right)$ for all $f_{r} \in \mathcal{Z}_{m, f, r}$.

(b) If $m$ is even, then $\sum_{f_{r} \in \mathcal{Z}_{m, f, r}} p\left(f_{r}\right)=0$.

We conclude this section by showing how Theorem 1.1a follows from Theorem 4.2.

Proof of Theorem 1.1a: Together with (4.16), Theorem 4.2 shows

$$
H_{m^{1}, \ldots, m^{k}}^{h, p}=\frac{1}{(d !)^{3} \prod_{i=1}^{k} m^{i} !} \sum_{m: o d d}|m|^{2} \sum_{f=\left(f_{1}, f_{e}, f_{2}\right) \in \mathcal{M}_{m, 0}} p\left(f_{1}\right) p\left(f_{2}\right)
$$

where the factor $|m|^{2}$ appears because the fiber $\mathcal{Z}_{m, f, r}(r \neq 0)$ consists of $|m|^{2}$ maps. Since the map (4.10) has degree $(m !)^{2}$, the last sum in (4.17) is $\sum_{f=\left(f_{1}, f_{e}, f_{2}\right) \in \mathcal{M}_{m, 0}} p\left(f_{1}\right) p\left(f_{2}\right)=\frac{1}{(m !)^{2}} \sum_{\left(f_{1}, f_{e}, f_{2}\right) \in \mathcal{P}_{m}} p\left(f_{1}\right) p\left(f_{2}\right)$

$$
\begin{aligned}
& =\frac{1}{(m !)^{2}} \sum_{f_{e} \in \mathcal{M}_{m}^{e}}\left(\sum_{f_{1} \in \mathcal{M}_{m}^{1}} p\left(f_{1}\right)\right) \cdot\left(\sum_{f_{2} \in \mathcal{M}_{m}^{2}} p\left(f_{2}\right)\right) \\
& =\frac{(d !)^{3} m !}{|m|} \prod_{i=1}^{k} m^{i} \cdot H_{m^{1}, \ldots, m^{k_{0}, m}}^{h_{1}, p_{1}} \cdot H_{m, m^{k_{0}+1}, \ldots, m^{k}}^{h_{2}, p_{2}}
\end{aligned}
$$

where the last equality holds by (4.3) and (4.8). Theorem 1.1a follows from Equations (4.17) and (4.18).

The proof of Theorem $1.1 \mathrm{~b}$ is identical to that of Theorem 1.1a except that one uses a smooth family of target curves $\mathcal{D} \rightarrow \Delta$ and a line bundle $\mathcal{L} \rightarrow \mathcal{D}$ satisfying:

- The general fiber $D_{r}(r \neq 0)$ is a smooth curve of genus $h \geq 1$ and $\left.\mathcal{L}\right|_{D_{r}}$ is a theta characteristic.

- The central fiber of $\mathcal{D} \rightarrow \Delta$ is a connected nodal curve $\bar{D} \cup E$ where $\bar{D}$ is a smooth genus $h-1$ curve that meets $E \cong \mathbb{P}^{1}$ at two points.

- $\mathcal{L}$ restricts to $\mathcal{O}(1)$ on $E$ and to a theta characteristic on $\bar{D}$ with $p\left(\left.\mathcal{L}\right|_{\bar{D}}\right) \equiv p\left(\left.\mathcal{L}\right|_{D_{r}}\right)$ for $r \neq 0$. 
Minor modifications to the arguments of this section and to the constructions and calculations in Sections 5 to 10, yield parity formulas analogous to Theorem 4.2, which leads to Theorem 1.1b.

\section{The algebraic family moduli space}

In this section, we construct a deformation of a map $f: C \rightarrow D_{0}$ from a nodal curve to a nodal spin curve. The deformation has many components, indexed by roots of unity. Each component is a curve $\mathcal{C} \rightarrow \Delta$ over the disk with smooth total space, with a map to a deformation $\mathcal{D} \rightarrow \Delta$ of $D_{0}$ and a bundle $\mathcal{N} \rightarrow \mathcal{C}$ whose restriction to each general fiber $C_{s}$ is a theta characteristic on $C_{s}$. In fact, there are many such bundles $\mathcal{N}$; we fix one that makes computations in later sections possible.

Throughout this section we fix, once and for all, a partition $m=\left(m_{1}, \ldots\right.$, $\left.m_{\ell}\right)$ of $d$, a map $f=\left(f_{1}, f_{e}, f_{2}\right): C \rightarrow D_{0}$ in $\mathcal{M}_{m, 0}$ where $\mathcal{M}_{m, 0}$ is the space (4.9), and the spin structure $(\mathcal{L}, \Phi)$ on $\rho: \mathcal{D} \rightarrow \Delta$ in (4.15). As in Section 4, $D_{0}$ is a nodal curve $D_{1} \cup E \cup D_{2}$ with exceptional component $E=\mathbb{P}^{1}$ and with nodes $p^{1} \in D_{1} \cap E$ and $p^{2} \in D_{2} \cap E$. The domain $C$ is a nodal curve $C=C_{1} \cup C_{e} \cup C_{2}$ with $2 \ell$ nodes where $\chi\left(C_{e}\right)=2 \ell$ such that for $i=1,2$ and $j=1, \ldots, \ell$

- $f^{-1}\left(p^{i}\right)$ consists of the $\ell$ nodes $p_{j}^{i} \in C_{i} \cap E_{j}$,

- $C_{i}$ is smooth and $f_{i}=\left.f\right|_{C_{i}}: C_{i} \rightarrow D_{i}$ has ramification index $m_{j}$ at the node $p_{j}^{i}$,

- $C_{e}$ is a disjoint union of $\ell$ rational curves $E_{j}, f_{e}=\left.f\right|_{C_{e}}$ and each restriction $\left.f\right|_{E_{j}}: E_{j} \rightarrow E$ has degree $m_{j}$ and ramification index $m_{j}$ at $p_{j}^{i}$.

For $i=1,2$, let $\mathcal{R}_{f_{i}}$ denote ramification divisor of $f_{i}$, and let $\mathcal{R}_{f_{i}}^{\text {ev }}$ be the divisor on $C_{i}$ consisting of the even ramification points:

$$
\mathcal{R}_{f_{i}}^{\mathrm{ev}}=\sum_{j \mid m_{j} \text { is even }} p_{j}^{i} .
$$

By Remark 4.1, $\left|\mathcal{R}_{f_{i}}\right|$ and $\left|\mathcal{R}_{f_{i}}^{\mathrm{ev}}\right|$ are both even. For $j=1, \ldots, \ell$, we set

$$
n_{j}=\frac{|m|}{m_{j}} .
$$

Let $Q_{m}$ denote the set of vectors of the form $\zeta=\left(\zeta_{1}^{1}, \zeta_{1}^{2}, \ldots, \zeta_{\ell}^{1}, \zeta_{\ell}^{2}\right)$ where $\zeta_{j}^{1}$ and $\zeta_{j}^{2}$ are $m_{j}$-th roots of unity. The following is a main result of this section. 
Theorem 5.1. Let $f=\left(f_{1}, f_{e}, f_{2}\right) \in \mathcal{M}_{m, 0}$ and $Q_{m}$ be as above. Then, for each vector $\zeta \in Q_{m}$, there exists a family of curves $\mathcal{C}_{\zeta} \rightarrow \Delta$, over a disk $\Delta$ (with parameter $s$ ) with smooth total space $\mathcal{C}_{\zeta}$, a holomorphic map $\mathcal{F}_{\zeta}: \mathcal{C}_{\zeta} \rightarrow$ $\mathcal{D}$ and a line bundle $\mathcal{N}_{\zeta}$ over $\mathcal{C}_{\zeta}$ satisfying:

(a) For $s \neq 0$, the fiber $C_{\zeta, s}$ is smooth and the restriction $N_{\zeta, s}=\left.\mathcal{N}_{\zeta}\right|_{C_{\zeta, s}}$ is a theta characteristic on $C_{\zeta, s}$ and the restriction map $f_{\zeta, s}=\left.\mathcal{F}\right|_{C_{\zeta, s}}$ has the associated parity $p\left(f_{\zeta, s}\right)=p\left(N_{\zeta, s}\right)$ such that the last sum in (4.16) is

$$
\sum_{f_{r} \in \mathcal{Z}_{m, f, r}} p\left(f_{r}\right)=\sum_{\zeta \in Q_{m}} p\left(f_{\zeta, s}\right) \quad \text { where } r=s^{|m|}
$$

(b) The central fiber $C_{\zeta, 0}$ is a nodal curve $C_{1} \cup\left(\cup_{j=1}^{\ell} \bar{E}_{j}\right) \cup C_{2}$ where, as in the figure at the end of this section, each $\bar{E}_{j}$ is a chain of rational curves with dual graph

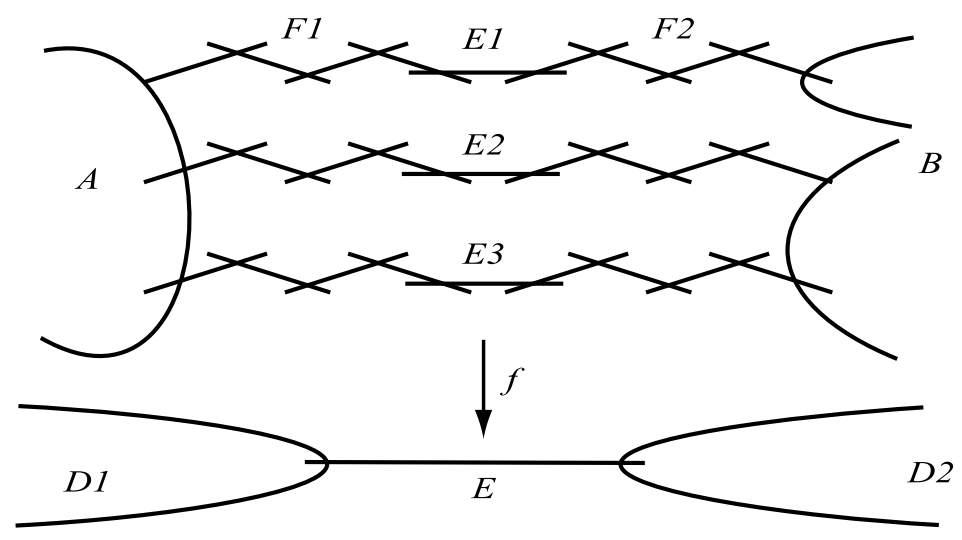

(c) $\left.\mathcal{N}_{\zeta}\right|_{C_{i}}=f_{i}^{*}\left(\left.\mathcal{L}\right|_{D_{i}}\right) \otimes \mathcal{O}\left(\frac{1}{2}\left(\mathcal{R}_{f_{i}}-\mathcal{R}_{f_{i}}^{e v}\right)\right)$ for $i=1,2$.

(d) $\left.\mathcal{N}_{\zeta}\right|_{E_{j ; k}^{i}}= \begin{cases}\mathcal{O}(1) & \text { if } m_{j} \text { is even and } k=n_{j}-1, \\ & \text { and if } m_{j} \text { is odd and } k=0 \\ \mathcal{O} & \text { otherwise. }\end{cases}$

Here, for the case $k=0, E_{j ; 0}^{1}=E_{j ; 0}^{2}$ denotes $E_{j}$. Note that $n_{j}>1$, whenever $m_{j}$ is even (because $\left|\mathcal{R}_{f_{i}}^{e v}\right|$ is even).

The proof of Theorem 5.1 requires six steps; each is a standard procedure in algebraic geometry. Steps 1 to 4 use Schiffer Variations (cf. [1]) and are described in detail in [9]. 
Step 1 - Deform the target: As in (4.5) there is an algebraic curve $\rho$ : $\mathcal{D} \rightarrow \Delta$ over the disk $\Delta$ with $k+3$ sections $Q^{i}$ whose central fiber is identified with $D_{0}$ with the marked points $q^{i}=Q^{i}(0)$. Denoting the coordinate on $\Delta$ by $r$, there are local coordinates $\left(u^{1}, v^{1}, r\right)$ and $\left(u^{2}, v^{2}, r\right)$ around the nodes $p^{1}$ and $p^{2}$ in $\mathcal{D}$ so that the fiber $D_{r}=\rho^{-1}(r)$ is locally given by $u^{1} v^{1}=r$ and $u^{2} v^{2}=r$.

Step 2 - Deform the domain: A similar construction yields a deformation $\varphi_{2 \ell}: \mathcal{X} \rightarrow \Delta_{2 \ell}$ of $C_{0}$ over polydisk

$$
\Delta_{2 \ell}=\left\{\mathbf{r}=\left(r_{1}^{1}, r_{1}^{2}, \ldots, r_{\ell}^{1}, r_{\ell}^{2}\right) \in \mathbb{C}^{2 \ell}:\left|r_{j}^{i}\right|<1\right\}
$$

Furthermore, there are local coordinates $\left(x_{j}^{i}, y_{j}^{i}, \mathbf{r}\right)$ around each node $p_{j}^{i}$ of $C_{0}$ in $\mathcal{C}$ in which the fiber $C_{\mathbf{r}}$ of $\rho$ over $r$ is given by $x_{j}^{i} y_{j}^{i}=r_{j}^{i}$.

Step 3 - Extend the map: The map $f: C \rightarrow D_{0}$ extends to a map of families over the curve $\mathcal{V} \subset \Delta_{2 \ell}$ defined by

$$
\mathcal{V}=\left\{\left(r_{1}^{1}\right)^{m_{1}}=\left(r_{1}^{2}\right)^{m_{1}}=\cdots=\left(r_{\ell}^{1}\right)^{m_{\ell}}=\left(r_{\ell}^{2}\right)^{m_{\ell}}=r \mid r \in \mathbb{C}\right\} .
$$

Near the nodes of $C_{0}$ the extension is given on $\varphi_{2 \ell}^{-1}(\mathcal{V})$ by

$$
\left(x_{j}^{i}, y_{j}^{i}, \mathbf{r}\right) \rightarrow\left(u^{i}, v^{i}, r\right), \quad \text { where } u^{i}=\left(x_{j}^{i}\right)^{m_{j}}, v^{i}=\left(y_{j}^{i}\right)^{m_{j}}, r=\left(r_{j}^{i}\right)^{m_{j}} .
$$

Note that this extension maps fibers to fibers only over $\mathcal{V}$.

Step 4 - Normalization: The one-dimensional variety (5.6) has $|m|^{2}$ branches at the origin. To separate the branches, we lift to the normalization as follows. For each vector $\zeta=\left(\zeta_{1}^{1}, \zeta_{1}^{2}, \ldots, \zeta_{\ell}^{1}, \zeta_{\ell}^{2}\right)$ in $Q_{m}$, define a holomorphic map

$$
\delta_{\zeta}: \Delta \rightarrow \Delta_{2 \ell} \quad \text { by } s \rightarrow\left(\zeta_{1}^{1} s^{n_{1}}, \zeta_{1}^{2} s^{n_{1}}, \zeta_{2}^{1} s^{n_{2}}, \zeta_{2}^{2} s^{n_{2}}, \ldots, \zeta_{\ell}^{1} s^{n_{\ell}}, \zeta_{\ell}^{2} s^{n_{\ell}}\right)
$$

where $n_{j}$ is the number (5.2). The pullback $\mathcal{X}_{\zeta}=\delta_{\zeta}^{*} \mathcal{X}$ is a deformation of $C$ over $\Delta$ :

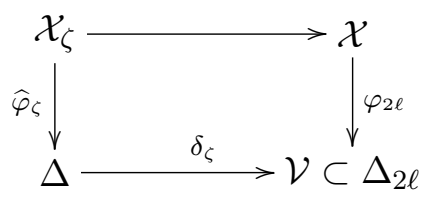

Near the node $p_{j}^{i}$ of the central fiber $C$, the fiber of $\mathcal{X}_{\zeta}$ over $s$ is the set of $\left(x_{j}^{i}, y_{j}^{i}, s\right) \in \mathbb{C}^{3}$ satisfying $x_{j}^{i} y_{j}^{i}=\zeta_{j}^{i} s^{n_{j}}$ and the pullback of (5.7) is a map 
$f_{\zeta}: \mathcal{X}_{\zeta} \rightarrow \mathcal{D}$ which, by (5.2), is given locally by

$$
G_{\zeta}\left(x_{j}^{i}, y_{j}^{i}, s\right)=\left(\left(x_{j}^{i}\right)^{m_{j}},\left(y_{j}^{i}\right)^{m_{j}}, s^{|m|}\right)
$$

Step 5 - Blow-up: The surface $\mathcal{X}_{\zeta}$ is singular at the nodes $p_{j}^{i}$ when $n_{j}>$ 1. The singularities can be resolved by repeatedly blowing up, as follows. Suppressing $i$ and $j$ from the notation, $\mathcal{X}_{\zeta}$ is locally given by $x y=\zeta s^{n_{j}}$ with $C_{1}$ given by $y=0$ and $E_{0}=E_{j}$ given by $x=0$.

FIRST BLOWUP: Blow up along the locus $y=s=0$ by setting $y=y_{1} s$ and pass to the proper transform. This introduces an exceptional curve $E_{1}$ on $C_{0}$ with coordinates $y_{1}$ and $x_{1}=1 / y_{1}$. The proper transform is given by

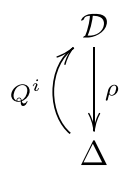

SECOND BLOWUP: Blow up along $y_{1}=s=0$ by setting $y_{1}=y_{2} s$. This introduces $E_{2}$ with coordinates $y_{2}$ and $x_{2}=1 / y_{2}$; the proper transform is given by

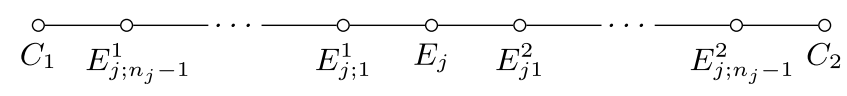

Blowing up $n_{j}-1$ times, and repeating on the other side of $E_{0}=E_{j}$ and at each node $p_{j}^{i}$, yields a smooth surface $\mathcal{C}_{\zeta}$ and a diagram

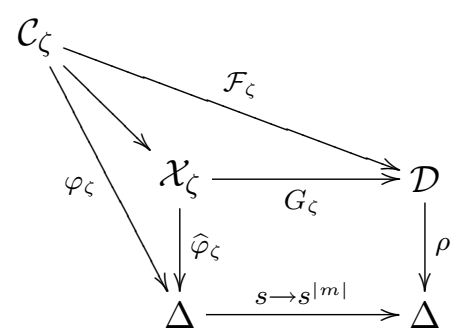

The central fiber of $\mathcal{C}_{\zeta} \rightarrow \Delta$ is as described in Theorem 5.1, and all other fibers are smooth. Using (5.8) and the equations $x=\zeta s^{n_{j}-n} x_{n}$ and $y=s^{n} y_{n}$, one sees that, for $1 \leq n<n_{j}$, the map $\mathcal{F}_{\zeta}: \mathcal{C}_{\zeta} \rightarrow \mathcal{D}$ is given locally near 
$E_{n} \cap E_{n-1}=\left\{y_{n-1}=s=0\right\} \cap\left\{x_{n}=s=0\right\}$ by

$$
\begin{aligned}
\mathcal{F}_{\zeta}\left(x_{n}, y_{n-1}, s\right)= & \left(\left(x_{n}\right)^{m_{j}\left(n_{j}-n+1\right)}\left(y_{n-1}\right)^{m_{j}\left(n_{j}-n\right)},\right. \\
& \left.\times\left(x_{n}\right)^{m_{j}(n-1)}\left(y_{n-1}\right)^{n m_{j}}, s^{|m|}\right)
\end{aligned}
$$

with $x_{n} y_{n-1}=s$ where $y_{0}=y$, and near $E_{n_{j}-1} \cap C_{1}$ by the same formula with $C_{1}$ and $x$ labeled as $E_{n_{j}}$ and $x_{n_{j}}$ and with $x y_{n_{j}-1}=\zeta s$.

We can now relate the fibers of $\mathcal{C}_{\zeta}$ to the spaces $\mathcal{Z}_{m, f, r}$ in (4.14). Note that for each vector $\zeta$ as in Step 4, the restriction of $\mathcal{F}_{\zeta}$ to the fiber over $r=s^{|m|} \neq 0$ is a map

$$
f_{\zeta, s}=\left.\mathcal{F}_{\zeta}\right|_{C_{\zeta, s}}: C_{\zeta, s} \rightarrow D_{r}
$$

Lemma 5.2. Whenever $s \neq 0$ and $r=s^{|m|}$, we have

$$
\mathcal{Z}_{m, f, r}=\bigcup_{\zeta \in Q_{m}}\left\{f_{\zeta, s}\right\}
$$

where the union is over all vectors $\zeta$.

Proof. Recall that $f: C \rightarrow D_{0}$ has contact marked points $q_{j}^{i}$ over $q^{i} \in D_{0}$ with multiplicities given by an odd partition $m^{i}$ for $1 \leq i \leq k+3$. By our choice of $q^{i}$ in Step 1, around each $q_{j}^{i}$ the map $\mathcal{F}_{\zeta}$ is

$$
(x, s) \rightarrow\left(f(x), s^{|m|}\right) .
$$

Hence, the pullback $\mathcal{F}_{\zeta}^{*} Q^{i}$ of $\mathcal{D} \rightarrow \Delta$ consists of $\ell\left(m^{i}\right)$ sections $Q_{j}^{i}$ given by $Q_{j}^{i}(s)=\left(q_{j}^{i}, s\right)$. After marking the points $Q_{j}^{i} \cap C_{\zeta, s}$, each of the $|m|$ maps (5.10) has contact marked points $Q_{j}^{i}(s)$ over $Q^{i}(r)$ with multiplicity $m_{j}^{i}$, and thus lies in the space $\mathcal{M}_{r}$ of (4.11). As $r=s^{|m|} \rightarrow 0$ we have $f_{\zeta, s} \rightarrow f$ in the Gromov topology; in particular, the stabilization of the domain $C_{\zeta, s}$ converges to $C$. The lemma follows because $\left|Q_{m}\right|=|m|^{2}=\left|\mathcal{Z}_{m, f, r}\right|$.

Step 6 - Twisting at nodes: The pullback $\mathcal{F}_{\zeta}^{*} \mathcal{L}$ of the spin structure $(\mathcal{L}, \Phi)$ on the family $\mathcal{D} \rightarrow \Delta$ is not a theta characteristic on the fibers of $\mathcal{C}$. In this step, we twist $\mathcal{F}_{\zeta}^{*} \mathcal{L}$ by a divisor $A$ to produce a line bundle

$$
\mathcal{N}_{\zeta}=\mathcal{F}_{\zeta}^{*} \mathcal{L} \otimes \mathcal{O}\left(\frac{1}{2} Q+A\right)
$$

over $\mathcal{C}_{\zeta}$ with the properties described in Theorem 5.1: it restricts to a theta characteristic on the general fiber, and is especially simple on the central chains $\bar{E}_{j}$. This twisting is crucial for later computations. 
Specifically, let $Q=\sum\left(m_{j}^{i}-1\right) Q_{j}^{i}$ be the divisor on $C_{\zeta}$ as above and let $A=\sum A_{j}$ where

$$
A_{j}= \begin{cases}\frac{n_{j} m_{j}-2}{2} E_{j}+\sum_{n=1}^{n_{j}-1} \frac{\left(n_{j}-n\right) m_{j}-2}{2}\left(E_{j ; n}^{1}+E_{j ; n}^{2}\right) & \text { if } m_{j} \text { is even } \\ \frac{n_{j}\left(m_{j}-1\right)}{2} E_{j}+\sum_{n=1}^{n_{j}-1} \frac{\left(n_{j}-n\right)\left(m_{j}-1\right)}{2}\left(E_{j ; n}^{1}+E_{j ; n}^{2}\right) & \text { if } m_{j} \text { is odd }\end{cases}
$$

To compute the restriction of $\mathcal{N}_{\zeta}$ to the fibers of $\mathcal{C}_{\zeta}$ we note a general fact: fix any irreducible component $\chi_{m}$ of $C_{0}$ and consider the bundle $\mathcal{O}\left(\chi_{m}\right)$ on $\mathcal{C}$. For each other component $\chi_{n}$, let $P_{m n}$ be the divisor $\chi_{m} \cap \chi_{n}$. By restricting local defining functions (cf. [5], page 253) one sees that the restriction of $\mathcal{O}\left(\chi_{m}\right)$ to a general fiber $C_{s}$ and to $\chi_{n}$ are:

$$
\begin{aligned}
\left.\mathcal{O}\left(\chi_{m}\right)\right|_{C_{s}} & =\mathcal{O},\left.\quad \mathcal{O}\left(\chi_{m}\right)\right|_{\chi_{n}}=\mathcal{O}\left(P_{m n}\right) \quad \text { for } m \neq n \\
\left.\mathcal{O}\left(\chi_{n}\right)\right|_{\chi_{n}} & =\mathcal{O}\left(-\sum_{m \neq n} P_{m n}\right) .
\end{aligned}
$$

Proof of Theorem 5.1. For each $\zeta$ and $s \neq 0$, the ramification divisor of the $\operatorname{map} f_{\zeta, s}$ in $(5.10)$ is $\left.Q\right|_{C_{\zeta, s}}$, and by (5.15) the restriction of $\mathcal{N}_{\zeta}$ to $C_{\zeta, s}$ is

$$
N_{\zeta, s}=f_{\zeta, s}^{*}\left(\left.\mathcal{L}\right|_{D_{r}}\right) \otimes \mathcal{O}\left(\left.\frac{1}{2} Q\right|_{C_{\zeta, s}}\right)
$$

Thus, as in (1.5), $N_{\zeta, s}$ is a theta characteristic on $C_{\zeta, s}$ and $f_{\zeta, s}$ has the associated parity $p\left(N_{\zeta, s}\right)$. Therefore (5.3) follows from Lemma 5.2. This completes the proof of part(a) of Theorem 5.1. Part(c) follows similarly, using (5.15) and noting that $f_{i}=\left.\mathcal{F}_{\zeta}\right|_{C_{i}}$ has ramification index $m_{j}$ at the node in $C_{i} \cap E_{j ; n_{j}-1}^{i}$. Part(b) was shown in Step 5 above. Finally, Part(d) follows by successively applying $(5.15)$, taking $\chi_{i}$ to be the various $E_{j ; n}^{i}$ and observing that $Q$ is disjoint from the chains $\bar{E}_{j}$ and that

- $\left.\mathcal{F}_{\zeta}^{*} \mathcal{L}\right|_{E_{j ; n}^{i}}=\mathcal{O}$ for $n=1, \ldots, n_{j}-1$ because the image $\mathcal{F}_{\zeta}\left(E_{j ; n}^{i}\right)$ is a point,

- $\left.\mathcal{F}_{\zeta}^{*} \mathcal{L}\right|_{E_{j}}=\mathcal{O}\left(m_{j}\right)$ since $\left.\mathcal{F}_{\zeta}\right|_{E_{j}}=\left.f\right|_{E_{j}}: E_{j} \rightarrow E$ has degree $m_{j}$ and $\left.\mathcal{L}\right|_{E}=\mathcal{O}(1)$. 


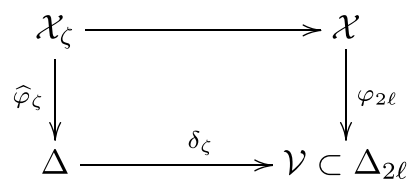

\section{The operators $L_{t}$ on the family}

For each $\zeta$, we now have an algebraic family $\mathcal{C}_{\zeta} \rightarrow \Delta$ and a bundle $\mathcal{N}_{\zeta}$ on $\mathcal{C}_{\zeta}$. One can then apply the construction of Section 2 to the fibers of $\mathcal{C}_{\zeta}$ to obtain operators

$$
L_{s, t}=\bar{\partial}_{C_{s}}+t R_{s}: \Omega^{0}\left(C_{\zeta, s}, N_{\zeta, s}\right) \rightarrow \Omega^{0,1}\left(C_{\zeta, s}, N_{\zeta, s}\right)
$$

that are a family version of the operators (2.4). This section describes a global construction on the complex surface $\mathcal{C}$ whose restriction to fibers gives the operators (6.1). The global construction will be important in later sections to obtain estimates on $L_{s, t}$ that are uniform in $s$.

Lemma 6.1. Each spin structure on $\mathcal{D}$ determines a nowhere-zero section $\psi$ of $K_{\mathcal{C}} \otimes\left(\mathcal{N}_{\zeta}^{*}\right)^{2} \otimes \mathcal{O}(-\hat{A})$ where, with the same notation as (5.14),

$$
\hat{A}=\sum_{j=1}^{\ell} \hat{A}_{j} \text { with } \hat{A}_{j}= \begin{cases}2 E_{j}+\sum_{n=1}^{n_{j}-1} 2\left(E_{j ; n}^{1}+E_{j ; n}^{2}\right) & \text { if } m_{j} \text { is even }, \\ n_{j} E_{j}+\sum_{n=1}^{n_{j}-1}\left(n_{j}-n\right)\left(E_{j ; n}^{1}+E_{j ; n}^{2}\right) & \text { if } m_{j} \text { is odd }\end{cases}
$$

Proof. The spin structure (4.15) on $\mathcal{D}$ vanishes to first order along $E \subset D_{0}$, so defines a section $\phi$ of $K_{\mathcal{D}} \otimes\left(\mathcal{L}^{*}\right)^{2} \otimes \mathcal{O}(-E)$. Noting that $\mathcal{O}\left(D_{0}\right)=\mathcal{O}$, we can write $\mathcal{O}(-E)$ as $\mathcal{O}\left(D_{1}+D_{2}\right)$. Using the definition (5.13), the pullback $\psi=\mathcal{F}_{\zeta}^{*} \phi$ is then a section of

$$
\mathcal{F}_{\zeta}^{*}\left(K_{\mathcal{D}}\right) \otimes \mathcal{O}(Q) \otimes \mathcal{O}(2 A) \otimes\left(\mathcal{N}_{\zeta}^{*}\right)^{2} \otimes \mathcal{F}_{\zeta}^{*} \mathcal{O}\left(D_{1}+D_{2}\right)
$$

Recall that the ramification divisor $\mathcal{R}_{\mathcal{F}_{\zeta}}$ of the map $\mathcal{F}_{\zeta}$ has local defining functions given by the Jacobian of $\mathcal{F}_{\zeta}$. One can thus see from (5.9) and (5.12) that $\mathcal{R}_{\mathcal{F}_{\zeta}}=Q+|m| C_{\zeta, 0}$. Choosing a trivialization $\mathcal{O}\left(C_{\zeta, 0}\right)=\mathcal{O}$, the 
Hurwitz formula gives

$$
K_{\mathcal{C}_{\zeta}}=\mathcal{F}^{*} K_{\mathcal{D}} \otimes \mathcal{O}\left(\mathcal{R}_{\mathcal{F}_{\zeta}}\right)=\mathcal{F}_{\zeta}^{*} K_{\mathcal{D}} \otimes \mathcal{O}(Q)
$$

From the second equation in (5.9) we also have

$$
\mathcal{F}_{\zeta}^{*} \mathcal{O}\left(D_{1}+D_{2}\right)=\mathcal{O}\left(|m| C_{1}+|m| C_{2}+\sum_{j=1}^{\ell} \sum_{n=1}^{n_{j}-1} n m_{j}\left(E_{j ; n}^{1}+E_{j ; n}^{2}\right)\right)
$$

because $\left\{v^{i}=0\right\} \subset D_{i}$ and $\left\{y_{n-1}^{i}=0\right\} \subset E_{j ; n}^{i}$. Together with the fact $\mathcal{O}\left(|m| C_{\zeta, 0}\right)=\mathcal{O}$, the last two displayed equations imply that the right-hand side of $(6.2)$ is $K_{\mathcal{C}} \otimes\left(\mathcal{N}_{\zeta}^{*}\right)^{2} \otimes \mathcal{O}(-\hat{A})$.

Corollary 6.2. There is a conjugate-linear bundle map $R_{\zeta}: \mathcal{N}_{\zeta} \rightarrow \bar{K}_{\mathcal{C}} \otimes$ $\mathcal{N}_{\zeta}$ whose divisor is $\hat{A}$.

Proof. Choose a global section $a$ of $\mathcal{O}(\hat{A})$ with divisor $\hat{A}$. Then with $\psi$ as in Lemma $6.1, \psi \otimes a$ is a section of $K_{\mathcal{C}} \otimes\left(\mathcal{N}^{*}\right)^{2}$ whose divisor is $\hat{A}$. Regarding this as a map $\hat{\psi}: \mathcal{N}_{\zeta} \rightarrow K_{\mathcal{C}} \otimes \mathcal{N}_{\zeta}^{*}$ and composing with the (conjugate-linear) star operator $\bar{*}: \Omega^{2,0}\left(\mathcal{C}, \mathcal{N}_{\zeta}^{*}\right) \rightarrow \Omega^{0,2}\left(\mathcal{C}, \mathcal{N}_{\zeta}\right)$ gives a bundle map

$$
R_{\zeta}=\bar{*} \hat{\psi}: \mathcal{N}_{\zeta} \rightarrow \bar{K}_{\mathcal{C}} \otimes \mathcal{N}_{\zeta}
$$

with divisor $\hat{A}$.

Because $\mathcal{C}$ is a smooth surface, the canonical bundle $K_{\mathcal{C}}$ is isomorphic to the relative dualizing sheaf $\omega_{\zeta}$ of $\varphi_{\zeta}: \mathcal{C} \rightarrow \Delta$. In fact, the restrictions of $K_{\mathcal{C}}$ and $\omega_{\zeta}$ are related by the commutative diagram

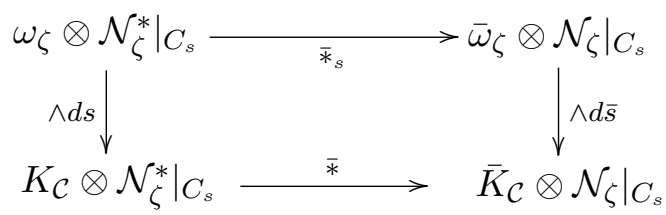

where $\bar{*}$ is as in Corollary $6.2, \bar{*}_{s}$ is the similar operator on the fiber $C_{s}$ of $\mathcal{C}$, and all four arrows are isomorphisms. In local coordinates $(x, y, s)$ near a node $x y=s$ of $C_{s}$, we have $d s=x d y+y d x$ and $\omega_{\zeta}$ is freely generated by $\tau=\frac{d x}{x}=-\frac{d y}{y}$. The star operator on $C_{s}$ is multiplication by $i$ on $(1,0)$ forms and by $-i$ on $(0,1)$-forms, so $\bar{*} \tau=* \bar{\tau}=-i \bar{\tau}$. The diagram commutes 
because, after restricting to $C_{s}$ and suppressing the bundle coordinates, $\tau \wedge$ $d s=\frac{d x}{x} \wedge(x d y+y d x)=d x \wedge d y$ and hence

$$
\bar{*}(\tau \wedge d s)=*(d \bar{x} \wedge d \bar{y})=-i *(d \bar{x} \wedge d \bar{y})=-i *(\bar{\tau} \wedge d \bar{s})=\left(\bar{*}_{s} \tau\right) \wedge d \bar{s} .
$$

Diagram (6.6) implies that for each $s$ there is a section $\psi_{s}$ of $\omega_{\zeta} \otimes \mathcal{N}_{\zeta}^{*}$ on $C_{\zeta, s}$ such that $\psi_{s} \wedge d s$ is the section $\hat{\psi}$ in (6.5). Consequently, for each $s$, $R_{s}=\bar{*}_{s} \psi_{s}$ is a conjugate-linear bundle map

$$
R_{s}: N_{\zeta, s} \rightarrow \bar{\omega}_{\zeta} \otimes N_{\zeta, s}
$$

between bundles on the curve $C_{\zeta, s}$. Let $N_{\zeta, i}=\left.\mathcal{N}_{\zeta}\right|_{C_{i}}$ for $i=1,2$.

Theorem 6.3. The map (6.7) satisfies Properties (2.2). Furthermore,

(a) On each smooth fiber $C_{\zeta, s}, R_{s}$ is an isomorphism $N_{\zeta, s} \rightarrow \bar{K}_{C_{\zeta, s}} \otimes N_{\zeta, s}$.

(b) For $i=1,2$, the restriction of $R_{0}$ to $C_{i}$ is a map $R_{i}: N_{\zeta, i} \rightarrow \bar{K}_{C_{i}} \otimes N_{\zeta, i}$ with divisor $\mathcal{R}_{f_{i}}^{e v}$.

Proof. The proof of Lemma 2.1 shows that $R_{s}$ satisfies Properties (2.2). By Diagram (6.6) we have $R_{s} \wedge d \bar{s}=\bar{*}_{s} \psi_{s} \wedge d \bar{s}=R_{\zeta}$, so the divisor of $R_{s}$ is $\hat{A} \cap C_{\zeta, s}$. Statement (a) holds because this intersection is empty for $s \neq 0$. For (b), note that the restriction of $\omega_{\zeta}$ to $C_{i}$ is $K_{C_{i}} \otimes \mathcal{O}\left(\sum_{j} p_{j}^{i}\right)$, so the divisor of $R_{s}$ is $C_{i} \cap \hat{A}-\sum_{j} p_{j}^{i}=\mathcal{R}_{f_{i}}^{e v}$.

It is useful to have a local formula for $R$ around the nodes $p_{j}^{i}$, where $C_{i}$ meets the chain $\bar{E}_{j}$. As in (5.9), we have local coordinates $(x, y, s)$ around $p_{j}^{i}$ in which $C_{1}=\{y=s=0\}$ and $E_{j, n_{j}-1}^{i}=\{x=s=0\}$. By Corollary 6.2 and the definition of $\hat{A}$, there is a local nowhere-zero section $\nu$ of $\mathcal{N}_{\zeta}$ and a constant $a \in \mathbb{C}^{*}$ such that $R(\nu)=a \bar{x}^{p} \bar{\tau} \otimes \nu$ where $p=2$ if $m_{j}$ is even and $p=1$ is $m_{j}$ is odd. By replacing $\nu$ by $e^{i \theta} \nu$, we can assume that $a$ is real and positive. Thus after writing $\tau$ as $d x / x$ we have

$$
\left.R(\nu)\right|_{\bar{E}_{j}}=\left.0 \quad R(\nu)\right|_{C_{i}}=\left\{\begin{array}{lll}
a \bar{x} & d \bar{x} \otimes \nu & m_{j} \text { even } \\
a & d \bar{x} \otimes \nu & m_{j} \text { odd }
\end{array}\right.
$$

for some real $a>0$. Similarly, one finds that at each interior nodes of $\bar{E}_{j}$, there are local coordinates in which $R(\nu)=a \bar{x} \bar{y}^{2} d \bar{x} \otimes \nu$.

We conclude this section by stating two facts about the index of the operators (6.1). 
Lemma 6.4. For $s \neq 0$, the operator $L_{s, t}$ on $C_{s}$ has index 0 , and for $i=1,2$ the restriction of $L_{0, t}$ to $C_{i}$ has index $-\ell^{e v}$ where $\ell^{e v}$ is the number of even ramification points of $f_{i}=\left.f_{0}\right|_{C_{i}}$.

Proof. For each $s, L_{s, t}$ is a compact perturbation of the $\bar{\partial}$-operator, so its index is twice of the holomorphic Euler characteristic $\chi\left(N_{\zeta, s}\right)$. But $\chi\left(N_{\zeta, s}\right)=$ 0 for $s \neq 0$ because $N_{\zeta, s}$ is a theta characteristic on $C_{s}$. Similarly, for $i=1,2$, $\left.\mathcal{N}\right|_{D_{i}}$ is a theta characteristic on $D_{i} \operatorname{so} 2 \operatorname{deg}\left(\left.\mathcal{N}\right|_{D_{i}}\right)=2 h-2$. Theorem $5.1 \mathrm{c}$, the Riemann-Roch and Riemann-Hurwitz formulas then give

$2 \chi\left(N_{\zeta, i}\right)=-\operatorname{deg}\left(f_{i}^{*} T D_{i}\right)+\operatorname{deg}\left(\mathcal{R}_{f_{i}}-\mathcal{R}_{f_{i}}^{\mathrm{ev}}\right)+\chi\left(C_{i}\right)=-\operatorname{deg}\left(\mathcal{R}_{f_{i}}^{\mathrm{ev}}\right)=-\ell^{\mathrm{ev}}$.

\section{Bundles of eigenspaces}

In Section 5, we constructed curves $\mathcal{C}_{\zeta} \rightarrow \Delta$ over the disk whose general fibers are smooth and whose central fiber $C_{0}$ is a union $C_{1} \cup E \cup C_{2}$ of nodal curves where $C_{1}$ and $C_{2}$ are disjoint and

$$
\bar{E}=\cup_{j} \bar{E}_{j}
$$

where each $\bar{E}_{j}$ is the chain of rational curves (5.4). For simplicity, we will drop $\zeta$ from our notation. There is also is a line bundle $\mathcal{N} \rightarrow \mathcal{C}$ whose restriction $N_{s}$ to each fiber $C_{s}$ comes with the bundle map $R_{s}$ described in Theorem 6.3 and the one-parameter family of operators

$$
L_{t}=\bar{\partial}+t R_{s}
$$

To take adjoints, we fix a Hermitian metric on $\mathcal{N}$ and a Riemannian metric $g$ on $\mathcal{C}$, with $g$ chosen to be Euclidean in the local coordinates $(x, y, s)$ around in node of $C_{0}$ (as described in Section 5).

On each curve $C_{s}$, the operator $L_{t}^{*} L_{t}$ on $N_{s}$ has non-negative real eigenvalues $\{\lambda\}$ that vary continuously with $s$ for $s \neq 0$. Given a function $\lambda_{1}(s)>$ 0 on $\Delta$ (we will fix a value later), consider the family of vector spaces $\mathcal{E} \rightarrow \Delta$ whose fiber over $s$ is spanned by the low eigensections as in (3.6):

$$
\mathcal{E}_{s}=\operatorname{span}_{\mathbb{R}}\left\{\xi \in L^{2}\left(C_{s} ; N_{s}\right) \mid L_{t}^{*} L_{t} \xi=\lambda \xi \text { for } \lambda<\lambda_{1}\right\}
$$

The eigensections of $L_{t} L_{t}^{*}$ give a similar family $\mathcal{F} \rightarrow \Delta$ of $L^{2}$ sections:

$$
\mathcal{F}_{s}=\operatorname{span}_{\mathbb{R}}\left\{\eta \in L^{2}\left(C_{s} ; \bar{K}_{C_{s}} \otimes N_{s}\right) \mid L_{t} L_{t}^{*} \eta=\lambda \eta \text { for } \lambda<\lambda_{1}\right\}
$$


and $L_{t}$ is a bounded finite-dimensional linear map $L_{t}: \mathcal{E}_{s} \rightarrow \mathcal{F}_{s}$. In general, the dimension of such eigenspaces can jump as $s$ varies. This section establishes conditions under which $\mathcal{E}$ and $\mathcal{F}$ are actually vector bundles over $\Delta$.

We will show that the spaces of $\mathcal{E}_{s}$ can be modeled on the space of holomorphic sections of $\mathcal{N}$ along the central fiber $C_{0}$.

Lemma 7.1. Let $\mathcal{E}_{0}=\left\{\right.$ continuous $\left.\psi \in H^{0}\left(C_{0}, N_{0}\right)\right\}$. There are $L^{2}$ orthogonal decompositions

$$
\mathcal{E}_{0}=W \oplus \mathcal{E}_{0}^{\prime} \quad W=\bigoplus_{j \mid m_{j} \text { even }} W_{j}
$$

where $W=\operatorname{ker} L_{t} \cap \mathcal{E}_{0}$, each $W_{j}$ is a 1-dimensional complex space and $\mathcal{E}_{0}^{\prime} \cong$ $H^{0}\left(C_{1}, N_{1}\right) \oplus H^{0}\left(C_{2}, N_{2}\right)$. Furthermore, $\mathcal{F}_{0}=$ ker $\left.L_{0, t}^{*}\right|_{C_{0}}$ has real dimension $2 \ell^{\mathrm{ev}}$.

Proof. Because $R$ is non-trivial on $C_{1} \cup C_{2}$ and trivial on $\bar{E}$, the proof of Theorem 2.2 shows that any continuous $\psi \in \operatorname{ker} L_{t}$ vanishes on $C_{1} \cup C_{2}$ and is holomorphic on $\bar{E}$, so lies in the direct sum of the $L^{2}$ orthogonal complex vector spaces

$$
W_{j}=\left\{\text { continuous } \psi \in H^{0}\left(C_{0}, N_{0}\right) \text { with support on } \bar{E}_{j}\right\}
$$

If $m_{j}$ is odd, $N_{0}$ is $\mathcal{O}(1)$ on the center component of $\bar{E}_{j}$ and is trivial the other irreducible components; the boundary conditions (7.4) then imply that $W_{j}=0$. If $m_{j}$ is even, $N_{0}$ is $\mathcal{O}(1)$ on the first and last components of $\bar{E}_{j}$ and trivial on the others; hence $W_{j} \cong \mathbb{C}$ and each $\psi \in W_{j}$ is constant on $\bar{E}_{j}$ except on the end components.

One similarly sees that each $\psi \in H=H^{0}\left(C_{1}, N_{1}\right) \oplus H^{0}\left(C_{2}, N_{2}\right)$ extends continuously and holomorphically over $C_{0}$; the extension is unique modulo $W$ and hence there is a unique extension $\bar{\psi}$ perpendicular to $W$. Let $\mathcal{E}_{0}^{\prime} \cong H$ denote the set of all extensions. Then for each continuous $\xi \in H^{0}\left(C_{0}, N_{0}\right)$ there is a $\bar{\psi} \in \mathcal{E}_{0}^{\prime}$ so that $\xi-\bar{\psi}$ has support in $\bar{E}$, and therefore lies in $W$ as above. Thus, $\mathcal{E}_{0}$ decomposes as in (7.3).

Finally, note that the restriction of each $\eta \in \mathcal{F}_{0}=\operatorname{ker} L_{0, t}^{*}$ to each component of $\bar{E}$ satisfies $\left(\bar{\partial}^{*}+t R^{*}\right) \eta=0$ with $R=0$, so by Theorem $5.1 \mathrm{~d}$ lies in $H^{01}\left(\mathbb{P}^{1}, \mathcal{O}\right)=0$ or $H^{01}\left(\mathbb{P}^{1}, \mathcal{O}(1)\right)=0$. Thus, $\eta=\eta_{1}+\eta_{2}$ where $\eta_{i}$ lies in the kernel of the operator $L_{i}=\left.L_{0, t}\right|_{C_{i}}$. But Theorem 2.2 and Lemma 6.4 
show that

$$
\operatorname{dim} \operatorname{ker} L_{i}^{*}=\operatorname{dim} \operatorname{ker} L_{i}-\operatorname{index} L_{i}=0-\left(-\ell^{\mathrm{ev}}\right)=\ell^{\mathrm{ev}} \text {, }
$$

so we conclude that $\mathcal{F}_{0}$ has real dimension $2 \ell^{\mathrm{ev}}$.

The following theorem shows that the decomposition Lemma 7.1 on the nodal curve $C_{0}$ carries over to nearby smooth curves. Parts (a) and (b) cover the case where $|t|$ is small, part (d) covers the case where $|t|$ is large, and (c) holds for all $t$. The upshot is that the low eigenspaces are of three types: one whose eigenvalues grow linearly with $t$, one whose eigenvalues are logarithmically small in $|s|$, and one whose eigenvalues are bounded by $|s|^{2}\left(1+t^{2}\right)$ and which splits as a sum of 2-dimensional eigenspaces.

$$
\lambda_{1}(s)=\frac{c_{0}}{|\log | s||}
$$

Theorem 7.2. (a) There is a $c_{0}>0$ such that, with $\lambda_{1}(s)$ as in (7.5) and $0<|s|,|t| \ll 1$, the low eigenspaces (7.1) and (7.2) form vector bundles $\mathcal{E}_{W}, \mathcal{E}^{\prime}$ and $\mathcal{F}^{\prime}$ over $\Delta$ and $\mathcal{F}^{0}$ over $\Delta \backslash\{0\}$ and a diagram of bundle maps

$$
\Delta \times\left(W \oplus \mathcal{E}_{0}^{\prime}\right) \stackrel{\Phi}{\cong} \mathcal{E}_{W} \oplus \mathcal{E}^{\prime}
$$

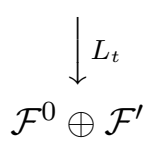

(b) There are positive constants $C_{1}, C_{2}, C_{3}$ such that for $t \neq 0$

$$
\begin{aligned}
\mathcal{E}_{W} & =\bigoplus\left\{\left.E_{\lambda}\left|\lambda \leq C_{1}\right| s\right|^{2}\left(1+t^{2}\right)\right\} \\
\mathcal{E}^{\prime} & =\bigoplus\left\{E_{\lambda} \mid C_{2} t^{2} \leq \lambda \leq C_{3}\left(|s|^{2}+t^{2}\right)\right\} .
\end{aligned}
$$

(c) For $t \neq 0$ and $|s| \ll 1+t^{2}$, the first component of $\Phi$ is a bundle isomorphism

$$
\Delta \times \bigoplus_{j} V_{j} \underset{\cong}{\stackrel{\Phi^{V}}{\longrightarrow}} \bigoplus_{j} \mathcal{E}_{j}
$$

where the $\mathcal{E}_{j}$ are real rank 2 bundles that are $L^{2}$ orthogonal up to terms of order $O\left(|s| \sqrt{1+t^{2}}\right)$.

(d) For each $\tau>0$ there is a $\delta>0$ such that (7.8) is an isomorphism onto the sum of the eigenspaces with eigenvalue $\lambda \leq C_{1}|s|^{2}\left(1+t^{2}\right)$, whenever $|t| \geq \tau$ and $|s|<\delta$. 
The proof of Theorem 7.2 occupies the rest of this section. The method is straightforward: transfer elements of ker $L_{t}$ on $C_{0}$ to $C_{s}$ by extending and cutting off, then estimate using the coordinates introduced in Section 5.

Proof. For each node $p$ of $C_{0}$, the construction of Section 5 provides coordinates $(x, y)$ on a ball $B(p, \varepsilon) \subset \mathcal{C}$ in which $C_{s}=\{x y=\zeta s\}$. After shrinking $\varepsilon$ we may assume these balls are disjoint and that on each ball there is a local holomorphic section $\nu$ of $\mathcal{N}$ with $\frac{1}{2} \leq|\nu|^{2} \leq 2$ pointwise. Let $B(\varepsilon)$ be the union of these balls. Each $\psi \in W \oplus \mathcal{E}_{0}^{\prime}$ is continuous and can be extended as follows:

- On $C_{0} \cap B(\varepsilon), \psi$ has the form $f \nu$ for some continuous holomorphic function. Extend this to the section $\psi^{\text {in }}=F \nu$ by setting

$$
F(x, y)=f(x, 0)+f(0, y)-f(0,0)
$$

on each $B(p, \varepsilon)$. This extension is continuous, holomorphic and agrees with $\psi$ along $C_{0}$.

- The construction of Section 5 shows that $C_{0} \backslash B(\varepsilon)$ is a disjoint union of embedded smooth curves. Hence we can extend $\psi$ to a smooth section $\psi^{\text {out }}$ of $\mathcal{N}$ on a neighborhood of $C_{0} \backslash B(\varepsilon)$ by parallel translation in the normal directions; the normal component of $\nabla \psi^{\text {out }}$ then vanishes along $C_{0}$.

To merge the above extensions, fix a smooth bump function $\beta_{\varepsilon}$ supported on $B(2 \varepsilon)$ with $\beta_{\varepsilon}=1$ on $B(\varepsilon)$ and with $0 \leq \beta_{\varepsilon} \leq 1$ and $\left|d \beta_{\varepsilon}\right| \leq 2 / \varepsilon$ everywhere. Then

$$
\widehat{\psi}=\beta_{\varepsilon} \psi^{\text {in }}+\left(1-\beta_{\varepsilon}\right) \psi^{\text {out }}
$$

is a smooth extension of $\psi$ to a section of $\mathcal{N}$ on a neighborhood of $C_{0}$. After choosing an $L^{2}$ orthonormal basis $\left\{\psi_{k}\right\}$ of $W \oplus \mathcal{E}_{0}^{\prime}$, this construction creates extensions $\left\{\widehat{\psi}_{k}\right\}$. We can then define a linear map $\Psi_{s}: W \oplus \mathcal{E}_{0} \rightarrow$ $C^{\infty}\left(C_{s}, N_{s}\right)$ for each small $s$ by setting

$$
\Psi_{s}\left(\psi_{k}\right)=\psi_{k, s} \quad \text { where } \psi_{k, s}=\left.\widehat{\psi}_{k}\right|_{C_{s}}
$$

for each basis vector $\psi_{k}$ and extending linearly. For each $j, \psi_{s}=\psi_{k, s}$ is continuous, holomorphic on $C_{s} \cap B(\varepsilon)$, and satisfies the following bounds for $|s|<1$ : 
(i) Because $\psi^{\text {in }}$ and $\psi^{\text {out }}$ are continuous extensions of $\psi$, we have $\mid \psi_{s}^{\text {in }}-$ $\psi_{s}^{\text {out }}\left|\leq c_{1}(\varepsilon)\right| s \mid$ on the region $A_{s}(\varepsilon)=C_{s} \cap(B(2 \varepsilon) \backslash B(\varepsilon))$, which contains the support of $d \beta_{\varepsilon}$.

(ii) On the complement of $B(\varepsilon)$, the curves $C_{s}$ converge to $C_{0}$ in $C^{1}$ as $s \rightarrow$ 0 and $\bar{\partial} \psi_{k, 0}=0$. Hence, $\left|\bar{\partial} \psi_{k, s}^{\text {out }}\right| \leq c_{2}(\varepsilon)|s|$ on the support of $1-\beta_{\varepsilon}$.

The $L^{2}$ norm of $L_{t} \psi_{s}=\bar{\partial} \beta_{\varepsilon}\left(\psi_{s}^{\text {in }}-\psi_{s}^{\text {out }}\right)+\left(1-\beta_{\varepsilon}\right) \bar{\partial} \psi_{s}^{\text {out }}+t R \psi_{k, s}$ therefore satisfies

$$
\begin{aligned}
\left\|L_{t} \psi_{k, s}\right\|^{2} & \leq c_{3}|s|^{2}\left(\int_{A_{s}(\varepsilon)} \frac{8}{\varepsilon^{2}}+\operatorname{Area}\left(C_{s}\right)\right)+c_{4} t^{2}\left\|\psi_{k, s}\right\|^{2} \\
& \leq c_{5}\left(|s|^{2}+t^{2}\right)\left\|\psi_{k, s}\right\|^{2}
\end{aligned}
$$

where the last inequality holds because $R$ is bounded and $\left\|\psi_{k, s}\right\| \rightarrow\left\|\psi_{k}\right\|=1$ as $s \rightarrow 0$.

If $\psi_{k} \in W$ then (7.11) can be strengthened. There is a basis $\left\{\psi_{j}\right\}$ of $W$ where the support of $\psi_{j}$ lies in an even chain $\bar{E}_{j}$ and $R=0$ along that chain; we therefore have $\left|R \psi_{j, s}\right| \leq c_{6}|s|\left|\psi_{j, s}\right|$ outside the $2 \varepsilon$-balls around the even nodes $p_{j}^{i}$. In those $2 \varepsilon$-balls, there are local coordinates $(x, y)$ in which $x y=\zeta s$ on $C_{s}$ and $R$ has the form (6.8) and $\psi_{k}=b y$ for some $b \in \mathbb{C}$ (cf. Theorem 5.1d). Therefore, $\left|R \psi_{j, s}\right| \leq c_{3}|\bar{x} \bar{y}|=c_{7}|s|$ and (7.11) becomes

$$
\left\|L_{t} \psi_{j, s}\right\|^{2} \leq c_{8}|s|^{2}\left(1+t^{2}\right)\left\|\psi_{j, s}\right\|^{2}
$$

The constant $c_{5}$ and $c_{8}$ can be taken independent of $j$ and $k$, and hence (7.11) holds for all $\psi \in \mathcal{E}_{0}$ and (7.12) holds for all $\psi \in W$.

We also have a lower bound on $\left\|R \psi_{s}\right\|$ for $\psi_{s} \in \mathcal{E}^{\prime}$. In this case, $\psi$ is holomorphic and is non-zero on an open set in $C_{1} \cup C_{2}$. The facts that $|R|$ is non-zero almost everywhere on $C_{i}$ and $\left\|\psi_{s}\right\| \rightarrow\left\|\psi_{k}\right\|=1$ as $s \rightarrow 0$ imply that, for small $|s|$,

$$
\left\|R \psi_{s}\right\|^{2} \geq \int_{C_{i} \backslash B(2 \varepsilon)} t^{2}|R|^{2}\left|\psi_{s}\right|^{2} \geq c_{9} t^{2} \int_{C_{i} \backslash B(2 \varepsilon)}\left|\psi_{s}\right|^{2} \geq c_{10} t^{2}\left\|\psi_{s}\right\|^{2}
$$

At this point we can define $\mathcal{E}$ and the decomposition $\mathcal{E}=\mathcal{E}_{W} \oplus \mathcal{E}^{\prime}$ by projecting onto low eigenspaces. For this, we assume that $s$ is not zero and is small enough that $|s|<c_{5}\left(|s|^{2}+t^{2}\right)<\frac{1}{2} \lambda_{1}(s)$ with $\lambda_{1}(s)$ as in (7.5). Applying Lemma 7.3 below twice shows that: 
- The composition $\Phi_{s}=\pi_{s} \Psi_{s}: \mathcal{E}_{0} \rightarrow \mathcal{E}_{s}$ of $\Psi_{s}$ with the $L^{2}$ orthogonal projection into the sum of the eigenspaces $E_{\lambda}$ on $C_{s}$ with $\lambda \leq c_{5}\left(|s|^{2}+\right.$ $\left.t^{2}\right)$ is an isometry up to terms of order $\left.O(|s|+|t|)\right)$.

- The composition $\Phi_{s}^{W}=\pi_{s} \Psi_{s}^{W}: W \rightarrow \mathcal{E}_{s}$ of $\Psi_{s}^{W}$ with the $L^{2}$ orthogonal projection into the sum $\mathcal{E}_{W}$ of the eigenspaces $E_{\lambda}$ on $C_{s}$ with $\lambda \leq c_{8}|s|^{2}\left(1+t^{2}\right)$ is an isometry up to terms of order $O\left(|s| \sqrt{1+t^{2}}\right)$; it has the form $\pi_{W} \Phi_{s}$ for small $|s|$ and $|t| \leq T$.

Because basis elements $\left\{\psi_{j}\right\}$ of $W=\oplus W_{j}$ have disjoint support, the image $\Phi^{W}\left(\oplus W_{j}\right)$ defines real rank 2 subbundles $\mathcal{E}_{j} \subset \mathcal{E}_{W}$ as in (7.8).

Now let $\mathcal{E}^{\prime}$ be the orthogonal complement of $\mathcal{E}_{W}$ in $\mathcal{E}$. Each eigenvector $\psi \in \mathcal{E}^{\prime}$ with eigenvalue $\lambda$ and norm 1 can be written as an orthogonal sum $\psi_{s}+v$ with $\psi_{s}$ in the image of (7.10) and $v \in \mathcal{E}_{W}$ satisfying $\|v\| \leq c_{8}(\sqrt{\ell(s)}+|t|)\left\|\psi_{s}\right\|$. We then obtain a lower bound on $\lambda=\left\|L_{t} \psi\right\|^{2}$ using (2.5), the inequality $2(a+b+c) \geq a^{2}-4 b^{2}-4 c^{2}$ and (7.13), noting that $R$ is bounded and $\psi$ has unit norm:

$$
\begin{aligned}
\lambda & \geq t^{2}\|R \psi\|^{2} \geq \frac{t^{2}}{2}\left[\left\|R \psi_{s}\right\|^{2}-4\|R w\|^{2}-4\|R v\|^{2}\right] \\
& \geq \frac{t^{2}}{4}\left[c_{11}-c_{12}\left(\ell(s)+|t|^{2}\right)\right] .
\end{aligned}
$$

For small $|s|$ and $|t|$, this gives the inequality $\lambda \geq C_{2} t^{2}$ in (7.7).

In fact, one can choose the constant $c_{0}$ in the definition (7.5) of $\lambda_{1}$ so that $\Phi_{s}: \mathcal{E}_{0} \rightarrow \mathcal{E}_{s}$ is surjective. The proof, which is crucial but rather technical, is given in the appendix.

To finish, set $\mathcal{F}^{0}=L_{t}\left(\mathcal{E}_{W}\right)$ and $\mathcal{F}^{\prime}=L_{t}\left(\mathcal{E}^{\prime}\right)$ and observe that $L_{t}$ maps the non-zero eigenspaces of $L_{t}^{*} L_{t}$ isomorphically to the eigenspaces of $L_{t} L_{t}^{*}$ with the same eigenvalues. But ker $L_{t}=0$ for $s \neq 0$ by Theorem 6.3 and ker $L_{t}=W$ on $C_{0}$ by Lemma 7.1 , so after shrinking $\Delta, \mathcal{F}^{\prime}$ is a bundle over $\Delta$ and $\mathcal{F}^{0}$ is a bundle over $\Delta \backslash\{0\}$. Finally, given $\tau>0$, we have $C_{1}|s|^{2}(1+$ $\left.\tau^{2}\right)<\min \left\{\lambda(s), C_{2} \tau^{2}\right\}$ for all small $|s|$; the eigenvalue bounds (7.7) then show that the sum of the eigenspaces in Theorem $7.2 \mathrm{~d}$ is exactly $\mathcal{E}_{W}$.

The proof of Theorem 7.2 made use of the following elementary lemma.

Lemma 7.3. Let $L: H \rightarrow H^{\prime}$ be a bounded linear map between Hilbert spaces so that all eigenvalues of $L^{*} L$ lie in $[0, \mu] \cup\left[\lambda_{1}, \infty\right)$ with $0<\mu<\lambda_{1}$. Consider the low eigenspace

$$
E_{\text {low }}=\bigoplus_{\lambda \leq \mu} E_{\lambda}
$$


and suppose that $V \subset H$ is a subspace with $|L v|^{2} \leq c \mu|v|^{2}$ for all $v \in V$. Then the orthogonal projection $\pi: V \rightarrow E_{\mathrm{low}}$ is the identity plus an operator of order $O(\sqrt{\mu})$.

Proof. Fix $v \in V$ and write $v=v_{0}+w$ where $v_{0}=\pi v$ and $\left\langle v_{0}, w\right\rangle=0$. Then $\left\langle L v_{0}, L w\right\rangle=\left\langle L^{*} L v_{0}, w\right\rangle$ vanishes because $L^{*} L v_{0} \in E_{\text {low }}$, while $|L w|^{2} \geq$ $\lambda_{1}|w|^{2}$ because $w \perp E_{\text {low }}$. Thus, $\lambda_{1}|w|^{2} \leq|L w|^{2}=|L v|^{2}-\left|L v_{0}\right|^{2} \leq c_{1} \mu|v|^{2}$, which means that $|v-\pi v|=|w| \leq c_{2} \sqrt{\mu}|v|$.

\section{Parity formulas}

As Section 7, we fix a partition $m$, a map $f=\left(f_{1}, f_{e}, f_{2}\right)$ in $\mathcal{M}_{m, 0}$ and $\zeta \in Q_{m}$; these data determine maps $f_{\zeta, s}: C_{\zeta, s} \rightarrow D_{s}$. Theorem 5.1 shows that for $s \neq 0$ the restriction of $\mathcal{N}$ is a theta characteristic $N_{s}$ on $C_{s}$, so defines a parity $p\left(f_{\zeta, s}\right)$. In fact, by Theorem $3.1, p\left(f_{\zeta, s}\right)$ is the TR spectral flow of the finite-dimensional linear map

$$
L_{s, t}=\bar{\partial}+t R_{s}: \mathcal{E}_{s} \rightarrow \mathcal{F}_{s}
$$

between the fibers of the bundle of Theorem 7.2. Moreover, this sign is independent of $s \neq 0$ and $t \neq 0$. In this section we will express the parity as a product of $2 \times 2$ determinants.

When the partition $m$ is odd, $f_{1}$ and $f_{2}$ themselves have parities given by the theta characteristics $N_{1}$ and $N_{2}$ on $C_{1}$ and $C_{2}$ (cf. Theorem 5.1), and these determine the parity of $f_{\zeta, s}$.

Lemma 8.1. If $m$ is odd then for every $\zeta \in Q_{m}$ and $s \neq 0$ the parity of $f_{\zeta, s}$ is

$$
p\left(f_{\zeta, s}\right)=p\left(f_{1}\right) \cdot p\left(f_{2}\right)
$$

Proof. If $m$ is odd, Lemma 7.1 shows that $W=0$ and the complex dimension of $\mathcal{E}_{0}$ is $h^{0}\left(N_{1}\right)+h^{0}\left(N_{2}\right)$. By the discussion in Section $3, p\left(f_{\zeta, s}\right)$ is $\operatorname{sgn} \operatorname{det} L_{s, t}: \mathcal{E}_{s}^{\prime} \rightarrow \mathcal{F}_{s}^{\prime}$, and this is independent of $s$ for small $|s|$ and $|t|$ in the trivialization of Theorem 7.2a. But for $s=0, L_{0, t}=\left.t R_{0}\right|_{\mathcal{E}_{0}}$ is a complex anti-linear isomorphism and therefore, as in (3.4),

$$
\operatorname{sgn} \operatorname{det} L_{0, t}=(-1)^{h^{0}\left(N_{1}\right)+h^{0}\left(N_{2}\right)}=p\left(f_{1}\right) \cdot p\left(f_{2}\right) .
$$

If $m$ is not an odd partition, the parity can be partially computed by the method of Lemma 8.1. 
Theorem 8.2. For each partition $m$ and $s \neq 0$, and for every $\zeta \in Q_{m}$ and $t \neq 0$, the parity of $f_{\zeta, s}$ is given by

$$
p\left(f_{\zeta, s}\right)=\left.(-1)^{h^{0}\left(N_{1}\right)+h^{0}\left(N_{2}\right)} \prod_{j \mid m_{j} \text { even }} \operatorname{sgn} \operatorname{det} L_{t}\right|_{\mathcal{E}_{j}} .
$$

Proof. Theorem 3.1 again shows that the parity is $\operatorname{sgn} \operatorname{det} L_{s, t}$ where $L_{s, t}$ is the map $L_{t}$ in Theorem 7.2 on the fiber over $s \neq 0$. Since $L_{t}$ preserves eigenspaces and ker $L_{t}=0$ for non-zero $s$ and $t$, we have

$$
p\left(f_{\zeta, s}\right)=\left.\left.\operatorname{sgn} \operatorname{det} L_{s, t}\right|_{\mathcal{E}_{s}^{\prime}} \cdot \operatorname{sgn} \operatorname{det} L_{s, t}\right|_{\mathcal{E}_{W}} .
$$

The first factor is equal to $p\left(f_{1}\right) p\left(f_{2}\right)$ as in the proof of Lemma 7.1. To decompose the second factor, choose an $L^{2}$ orthonormal basis of $\mathcal{E}_{W}$ consisting of eigenvectors $\psi_{j}^{i} \in \mathcal{E}_{j}$ of $L_{s, t}^{*} L_{s, t}$ with eigenvalues $\lambda_{j}^{i}$. Then $\left\|L_{s, t} \psi_{j}^{i}\right\|^{2}=\lambda_{j}^{i}$, while Theorem $7.2 \mathrm{c}$ gives

$$
\left|\left\langle L \psi_{j}^{i}, L \psi_{j^{\prime}}^{i}\right\rangle\right|=\left|\left\langle L^{*} L \psi_{j}^{i}, \psi_{j^{\prime}}^{i}\right\rangle\right|=\lambda_{j}^{i}\left|\left\langle\psi_{j}^{i}, \psi_{j^{\prime}}^{i^{\prime}}\right\rangle\right| \leq c_{1}|s| \sqrt{1+t^{2}} \lambda_{j}^{i}
$$

whenever $j^{\prime} \neq j$. Thus for fixed $t$ and $0<|s| \ll t$, the matrix of $L_{s, t}$ on $\mathcal{E}_{W}$ has a block form whose off-diagonal entries that are arbitrarily small compared to the diagonal entries, giving (8.1).

We conclude this section by observing that (8.1) remains valid when $L_{t}$ is replaced by a perturbation of the form $\hat{L}_{t}=L_{t}+\varepsilon t S$ for certain $S$. Specifically, applying Theorem 2.2 and the inequality $2 t|(\bar{\partial} \xi, S \xi)| \leq|\bar{\partial} \xi|^{2}+$ $t^{2}|S \xi|^{2}$, we have

$$
\begin{aligned}
\int_{C_{\zeta, s}}\left|\hat{L}_{t} \xi\right|^{2} & =\int_{C_{s}}\left|L_{t} \xi\right|^{2}+2 t \varepsilon(\bar{\partial} \xi, S \xi)+\varepsilon^{2} t^{2}|S \xi|^{2} \\
& \geq \int_{C_{\zeta, s}}(1-\varepsilon)|\bar{\partial} \xi|^{2}+t^{2}\left(|R \xi|^{2}-\varepsilon|S \xi|^{2}\right)
\end{aligned}
$$

Now recall from (6.8) that $R$ has the local expansion $R(\nu)=a \bar{x} d \bar{x} \nu$ at each even node $p=p_{j}^{i}$. Take $S$ of the same form: $S(\nu)=b \bar{x} d \bar{x} \nu$ near $p$ and bumped down to 0 outside a small neighborhood of $p$. Then there are constants $c_{1}, c_{2}$ such that

$$
|S \xi|^{2} \leq c_{1} r^{2}|\xi|^{2} \leq c_{2}|R \xi|^{2}
$$

Substituting into (8.2) shows that there is an $\varepsilon_{0}$ such that ker $\hat{L}_{t}=0$ for all $\varepsilon \leq \varepsilon_{0}$. This means that $\operatorname{sgn} \operatorname{det} \hat{L}_{t}=\operatorname{sgn} \operatorname{det} L_{t}$, so Proposition 7.3 holds 
with $R$ replaced by

$$
(R+\varepsilon S)(\nu)=(1+\varepsilon b) \bar{x} d \bar{x} \nu+\cdots
$$

for small $\varepsilon$. In this sense, we are free to replace the leading coefficient in the Taylor expansion of $R$ by any small perturbation and still have formula (8.1).

\section{Concentrating eigensections}

The last factor in the parity formula (8.1) is independent of non-zero $s$ and $t$. In this and the next section, we explicitly evaluate (8.1) by first taking $t$ large, and then $s$ small. The key observation is that as $t \rightarrow \infty$ the elements of ker $L_{t}^{*}$ on $C_{0}$ concentrate around the points where $R$ vanishes, and that on nearby smooth curves $C_{s}$ the low eigensections of $L_{t}^{*} L_{t}$ similarly concentrate with essentially explicit formulas.

On each smooth curve $C_{s}$, the adjoint of $L_{t}$ is the map $L_{t}^{*}: \Omega^{0,1}\left(N_{s}\right) \rightarrow$ $\Omega^{0}\left(N_{s}\right)$ given by

$$
L_{t}^{*}=\bar{\partial}^{*}+t R^{*}
$$

where $R^{*}$ (the pointwise adjoint of $R$ ) is a real bundle map that satisfies $R^{*} J=-J R^{*}$. Thus, $R^{*}$ is zero at those points where $R=0$, and is an isomorphism at all other points of $C_{s}$.

Lemma 9.1. $A=\bar{\partial} R^{*}+R \bar{\partial}^{*}$ is a bundle endomorphism and for each $s \neq 0$

$$
\int_{C_{s}}\left|L_{t}^{*} \eta\right|^{2}=\int_{C_{s}}\left|\bar{\partial}_{t}^{*} \eta\right|^{2}+t\langle\eta, A \eta\rangle+t^{2}\left|R^{*} \eta\right|^{2} \quad \forall \eta \in \Omega^{0,1}\left(C_{s}, N_{s}\right) .
$$

Proof. Formula (9.2) follows immediately from (9.1). Clearly $A$ is a first order linear differential operator, so is a bundle endomorphism if its symbol is 0 . For a non-zero tangent vector $v$, the symbols $\sigma_{v}$ of $\bar{\partial}$ and $-\sigma_{v}^{*}$ of $\bar{\partial}^{*}$ are isomorphisms, in fact, $\sigma_{v} \sigma_{v}^{*}=|v|^{2} \mathrm{Id}$. Taking the symbol of Equation $(2.2 \mathrm{c})$ gives $R^{*} \sigma_{v}=\sigma_{v}^{*} R$. But then $-|v|^{2}$ times the symbol is $A$ is

$$
-|v|^{2}\left(\sigma_{v} R^{*}-R \sigma_{v}^{*}\right)=\sigma_{v} R^{*} \sigma_{v} \sigma_{v}^{*}-\sigma_{v} \sigma_{v}^{*} R \sigma_{v}^{*}=\sigma_{v}\left[R^{*} \sigma_{v}-\sigma_{v}^{*} R\right] \sigma_{v}^{*}=0 .
$$

Lemma 9.2. For each neighborhood $\mathcal{B}$ of the set of zeros of $R^{*}$ there is a constant $c>0$ such that for all $t \geq 1$ each solution of $L_{s}^{*} L_{t} \eta=\lambda \eta$ with 
$\lambda \leq 1$ satisfies

$$
\int_{C \backslash \mathcal{B}}|\eta|^{2} \leq \frac{c}{t} \int_{C}|\eta|^{2} .
$$

Proof. Noting that $R^{*}$ is an isomorphism on $C \backslash \mathcal{B}$ and applying (9.2) gives the inequalities

$$
\begin{aligned}
\int_{C \backslash \mathcal{B}}|\eta|^{2} & \leq \frac{c}{t^{2}} \int_{C \backslash \mathcal{B}} t^{2}\left|R^{*} \eta\right|^{2} \leq \frac{c}{t^{2}} \int_{C}\left|L_{t}^{*} \eta\right|^{2}+t|\langle\eta, A \eta\rangle| \\
& \leq\left(\frac{c \lambda}{t^{2}}+\frac{c}{t}\|A\|_{\infty}\right) \int_{C}|\eta|^{2} .
\end{aligned}
$$

Lemma 9.2 means that as $t \rightarrow \infty$ the low eigensections of $L_{t}^{*} L_{t}$ concentrate in small neighborhoods $D(\varepsilon)$ of the zeros of $R^{*}$. The zeros occur only at the nodes with even multiplicity, where $R$ is given by (6.8). In particular, the elements of ker $L_{t}^{*}$ on $C_{0}$ concentrate at the even nodes $p_{j}^{i}$; these are explicitly described in the next lemma.

Writing $\eta=\phi d \bar{x} \otimes \nu$ in the coordinates of (6.8), the equation $L_{t}^{*} \eta=0$ takes the form

$$
-\frac{\partial \phi}{d x}+a t \bar{x} \bar{\phi}=0
$$

with $a>0$. Regarded as an equation on $\mathbb{C}$, this has the explicit $L^{2}$-normalized solution

$$
\eta=\phi d \bar{x} \otimes \nu, \quad \text { where } \phi(x)=i \sqrt{\frac{a t}{\pi}} \mathrm{e}^{-a t x \bar{x}}
$$

By cutting off and gluing, these forms give approximate elements of ker $L_{t}^{*}$ on curves. For example, we can glue onto $C_{1}$ as follows. Fix disjoint disks $D_{j}=D\left(p_{j}^{1}, 2 \varepsilon\right)$ in $C_{1}$ with coordinate $x$ centered on the points $p_{j}^{1}$ of even multiplicity. Choose a cutoff function $\beta_{j}=\beta_{\varepsilon}$ on $D_{j}$ as defined before (7.9) and set

$$
\mathcal{F}_{t}^{\text {approx }}=\operatorname{span}_{\mathbb{R}}\left\{\eta_{j}=\beta_{j} \cdot \phi(x) d \bar{x} \otimes \nu \mid j=1, \ldots, \ell^{\text {ev }}\right\}
$$

Lemma 9.3. For large $t$, the $L^{2}$ orthogonal projection $\pi_{a}: \mathcal{F}_{0, t}^{\text {approx }} \rightarrow$ ker $L_{t}^{*}$ on $C_{1}$ is an isomorphism and an isometry up to terms of order $O(1 / t)$. 
Proof. Integration in polar coordinates shows that $\frac{1}{2} \leq\left\|\eta_{j}\right\| \leq 2$ for all $j$ and all large $t$. Also, $L_{t}^{*} \eta_{j}=\left(\bar{\partial}+t R^{*}\right)\left(\beta_{j} \eta\right)=\beta_{j} L_{t}^{*} \eta-*(\bar{\partial} \beta \wedge * \eta)$ with $L_{t}^{*} \eta=0$. Integrating using (9.4) yields

$$
\left\|L_{t}^{*} \eta_{j}\right\|^{2} \leq \int_{D_{j}}|d \beta|^{2}|\eta|^{2} \leq \frac{c_{1}}{\varepsilon^{2}} \int_{\varepsilon}^{2 \varepsilon} \phi^{2}(r) r d r \leq \frac{c_{2}}{t^{2}}\left\|\eta_{j}\right\|^{2}
$$

after noting that $t^{2} \mathrm{e}^{-2 a t \varepsilon^{2}} \leq \varepsilon^{2}$ for large $t$. Lemma 7.3 then shows that $\pi_{a}$ is an isometry up to terms of order $1 / t$. It is an isomorphism because the $\left\{\eta_{j}\right\}$ are linearly independent (they have disjoint support) and ker $L_{t}^{*}$ and $\mathcal{F}_{0, t}^{\text {approx }}$ have the same dimension $\ell^{\text {ev }}$ by Lemma 7.1.

Lemma 9.3 is easily modified to apply to the smooth fibers $C_{s}$ of $\mathcal{C} \rightarrow \Delta$. For each node $p_{j}^{i}$ of $C_{0}$ with even multiplicity, let $\beta_{j}^{i}$ to be the function $\beta_{\varepsilon}$ as in (7.9) in $(x, y)$ coordinates on the ball $B\left(p_{j}^{i}, 2 \varepsilon\right)$ in $\mathcal{C}$ and replace (9.5) by the $2 \ell^{\mathrm{ev}}$-dimensional real vector space

$$
\mathcal{F}_{t}^{\text {approx }}=\operatorname{span}_{\mathbb{R}}\left\{\eta_{j}^{i}=\beta_{j}^{i} \cdot \phi(x) d \bar{x} \otimes \nu \mid j=1, \ldots, \ell^{\mathrm{ev}}, i=1,2\right\} .
$$

The restriction to $C_{s}$ followed by the $L^{2}$ orthogonal projection gives a linear map $\pi_{a}: \mathcal{F}_{t}^{\text {approx }} \rightarrow \mathcal{F}_{t}^{\text {low }}$ onto the low eigenspace of $L_{t} L_{t}^{*}$.

Theorem 9.4. Whenever $0<|s| \leq 1 / t^{2}$ and $t$ is large, $\pi_{a}: \mathcal{F}_{t}^{\text {approx }} \rightarrow \mathcal{F}_{t}^{\text {low }}$ is an isomorphism and an isometry up to terms of order $O(1 / t)$.

Proof. For each $i, j$, the support of $\eta_{j}^{i}$ lies in the portion of $C_{s}$ given by $(x, \zeta s / x)$ for $|s| / 2 \varepsilon \leq|x| \leq 2 \varepsilon$ with metric (A.3). Integration in polar coordinates shows that $\frac{1}{2} \leq\left\|\eta_{j}^{i}\right\| \leq 2$ for all large $t$. Noting that the support of $d \beta_{\varepsilon}$ lies in $A \cup A^{\prime}$ where $A=\{\varepsilon \leq r \leq 2 \varepsilon\}$ and $A^{\prime}=\{|s| \leq 2 \varepsilon r \leq 2|s|\}$. Then the $L^{2}$ norm of $L_{t}^{*} \eta$ is bounded by the first integral in (9.6) with the domain $D_{j}$ replaced by $A \cup A^{\prime}$. On $A$, the metric (A.3) approaches the Eucidean metric as $s \rightarrow 0$, so the bound (9.6) holds. On $A^{\prime}$, we can replace the conformally invariant quantity $|d \bar{x}|^{2} d \mathrm{vol}_{s}$ by its value in the Eucidean metric, namely $2 r d r d \theta$ and replace $\left|d \beta_{\varepsilon}\right|^{2}$ by its euclidean value times $\gamma^{-1}$. Noting that $\left|d \beta_{\varepsilon}\right|^{2} \gamma^{-1} \leq 4|\varepsilon s|^{-2}\left(1+|s|^{2} r^{-4}\right)^{-1} \leq c_{1} \varepsilon^{-2}$ on $A^{\prime}$ we have, as in (9.6),

$$
\int_{A^{\prime}}\left|d \beta_{\varepsilon}\right|^{2}\left|\eta_{j}^{i}\right|^{2} d \operatorname{vol}_{s} \leq \frac{c_{2}}{\varepsilon^{2}} \int_{\frac{|s|}{2 \varepsilon}}^{\frac{|s|}{\varepsilon}} \mathrm{e}^{-2 a t r^{2}} r d r \leq c_{3} \frac{t|s|^{2}}{\varepsilon^{4}} \leq \frac{c_{4}}{t^{2}}
$$


where we have used the inequalities $|s| \leq 1 / t^{2}$ and $\mathrm{e}^{-x}-\mathrm{e}^{-4 x} \leq 4 x$ for small $x$ and assumed that $t \geq \varepsilon^{-4}$. Combining these bounds yields

$$
\left\|L_{t}^{*} \eta_{j}^{i}\right\|^{2} \leq \frac{c_{5}}{t^{2}}\left\|\eta_{j}^{i}\right\|^{2}
$$

Lemma 7.3 then shows that $\pi_{a}$ is an isometry up to $O(1 / t)$ terms. It is an isomorphism because (7.6) implies that for $s \neq 0 \mathcal{F}_{t}^{\text {low }} \cong \mathcal{E}_{W} \cong W$ has real dimension $2 \ell^{\mathrm{ev}}$.

\section{Cancellation for even partitions}

For each partition $m$ and each $\zeta \in Q_{m}$, Theorem 8.2 expresses the parity $p\left(f_{\zeta, s}\right)$ in terms of the linear operators $L_{t, j}$ between the low eigenspaces $\mathcal{E}_{j}^{\text {low }}$ and $\mathcal{F}_{t, j}$ described in Theorem 7.2 and, for large $t$, Theorem 9.4. In this section we will use the concentration principle of Section 9 to show the following remarkable cancellation property.

Theorem 10.1. Let $m$ be an even partition as above and $s \neq 0$. Then

$$
\sum_{\zeta \in Q_{m}} p\left(f_{\zeta, s}\right)=0
$$

To prove Theorem 10.1, fix an even partition $m=\left(m_{1}, \ldots, m_{\ell}\right)$ and $\zeta=\left(\zeta_{1}^{1}, \zeta_{1}^{2}, \ldots, \zeta_{\ell}^{1}, \zeta_{\ell}^{2}\right)$ in $Q_{m}$ and choose an even component $m_{j}$ of $m$. We will focus on the chain $\bar{E}_{j}$ corresponding to the chosen $m_{j}$ and the nodal points $p=p_{j}^{1} \in C_{1} \cap \bar{E}_{j}$ and $q=p_{j}^{2} \in C_{2} \cap \bar{E}_{j}$ at the two ends of $\bar{E}_{j}$. For any bases $\left\{\psi_{1}, i \psi_{1}\right\}$ of $\mathcal{E}_{j}^{\text {low }}$ and $\left\{\eta_{1}, \eta_{2}\right\}$ of $\mathcal{F}_{j}^{\text {low }}$ the $j$ th factor in (8.1) is the sign of the determinant of the matrix

$$
L_{t, j}=\left.L_{t}\right|_{\mathcal{E}_{j}^{\text {low }}}=\left(\begin{array}{cc}
\left(\eta_{1}, L_{t} \psi_{1}\right) & \left(\eta_{2}, L_{t} \psi_{1}\right) \\
\left(\eta_{1}, L_{t} \psi_{2}\right) & \left(\eta_{2}, L_{t} \psi_{2}\right)
\end{array}\right)
$$

whose entries are given by conformally invariant $L^{2}$ inner products

$$
(\eta, \xi)=\int_{C_{\zeta, s}} \operatorname{Re}(\eta \wedge * \bar{\xi}) \eta, \quad \xi \in \Omega^{0,1}\left(C_{s}, N_{s}\right)
$$

on smooth fibers $C_{\zeta, s}$ of $\mathcal{C}_{\zeta}$. Theorems 7.2 and 9.4 give explicit formulas for sections $\psi_{j}$ and $\eta_{k}$ which give bases up to terms of order $O(\sqrt{|s|})$; using these in (10.1) will correctly give sgn det $L_{t, j}$ for small $s$.

The results of Section 9 show that for large $t$ the inner products in the first column of (10.1) are concentrated near $p$, and those in the second 
column are concentrated near $q$. Thus, det $L_{t, j}$ can be regarded as the contribution of an "instanton" tunneling across the chain $\bar{E}_{j}$ between $p$ and $q$.

To proceed, we need coordinate formulas for $\psi, \eta_{1}$ and $\eta_{2}$. Recall that there are local coordinates $(x, y)$ and a local holomorphic section $\nu$ of $\mathcal{N}$ defined a ball $B\left(p_{j}^{1}, 2 \varepsilon\right)$ so that $C_{\zeta, s}$ is locally given by $x y=\zeta s,|\nu(p)|=1$, and

$$
R(\nu)=a \bar{x} d \bar{x} \otimes \nu
$$

for a positive real constant $a$ (cf. (6.8)). Noting that elements in $W_{j}$ vanish to order 1 at $p$ and $q$, we can take $\psi_{1}$ and $\eta_{1}$ to be the restrictions of

$$
\psi=\beta(r) b y \nu \quad \eta=\frac{i}{2 \pi} \beta(\rho) \mathrm{e}^{-a t r^{2}} d \bar{x} \otimes \nu
$$

to $C_{\zeta, s}$ where $b \in \mathbb{C}^{*}, r=|x|, \rho^{2}=|x|^{2}+|y|^{2}$ as described in (7.10) and (9.4) but with $\eta$ normalized so that its $L^{2}$ norm satisfies $\|\eta\|^{2} \approx(4 \pi a t)^{-1}$ for large $t$.

Lemma 10.2. There is a $T$ such that whenever $t>T$ and $0<|s| \leq 1 / t$ we have

$$
\left(\eta, L_{t} \psi\right)_{C_{\zeta, s}}=a \operatorname{Re}(i b s \zeta) \mathrm{e}^{-a t|s|^{2} / 4 \varepsilon^{2}}+O\left(\frac{1}{\sqrt{t}}\right)
$$

Proof. Writing $L_{t} \psi=\bar{\partial} \psi+t R \psi$ with $R \psi=\beta \bar{b} \bar{y} R(\nu)=\beta \bar{b} a \overline{x y} d \bar{x} \otimes \nu$ and using the equation $x y=\zeta s$, one sees that the $L^{2}$ inner product is

$$
\left(\eta, L_{t} \psi\right)_{C_{\zeta, s}}=I+\frac{a t}{2 \pi} \operatorname{Re}(i b s \zeta) \int_{C_{\zeta, s}} \beta(\rho) \beta(r) \mathrm{e}^{-a t r^{2}}|d \bar{x}|^{2}|\nu|^{2} d \operatorname{vol}_{s}
$$

with $|I| \leq\|\eta\| \cdot\|\bar{\partial} \psi\| \leq c_{1}|s| / t \leq c_{1} / \sqrt{t}$ by (7.11), our normalization of $\eta$ and the hypothesis on $s$. As in the proof of Theorem 9.4, we can replace $|d \bar{x}|^{2} d \operatorname{vol}_{s}$ by $2 r d r d \theta$. Writing $|\nu|^{2}=1+h_{1}$ with $\left|h_{1}\right| \leq c_{2} r$ and integrating over $\theta$ gives

$$
\left(\eta, L_{t} \psi\right)_{C_{\zeta, s}}=2 a t \operatorname{Re}(i b s \zeta) \int_{|s| / 2 \varepsilon}^{\infty}\left(1+(\beta-1)+h_{2}\right) \mathrm{e}^{-a t r^{2}} r d r+O\left(\frac{1}{\sqrt{t}}\right)
$$

where $\beta=\beta(\rho) \beta(r)$ satisfies $|\beta-1| \leq 1$ and $\left|h_{2}\right| \leq c_{3} r$. The first and the last parts of this integral can be estimated using the formulas

$$
\int_{|s| / \varepsilon}^{\infty} \mathrm{e}^{-a t r^{2}} r d r=\frac{1}{2 a t} \mathrm{e}^{-a t|s|^{2} / 4 \varepsilon^{2}}, \quad \int_{0}^{\infty} r^{2} \mathrm{e}^{-a t r^{2}} d r=\frac{\sqrt{\pi}}{4}(a t)^{-3 / 2} .
$$


Noting that $\beta-1=0$ for $|s| / \varepsilon \leq r \leq \varepsilon$ and estimating as in (9.7), the middle integral is dominated by

$$
\begin{aligned}
& \int_{|s| / 2 \varepsilon}^{|s| / \varepsilon} \mathrm{e}^{-a t r^{2}} r d r+\int_{\varepsilon}^{\infty} \mathrm{e}^{-a t r^{2}} r d r \leq \frac{-1}{2 a t}\left[\left.\mathrm{e}^{-a t r^{2}}\right|_{|s| / 2 \varepsilon} ^{|s| / \varepsilon}+\mathrm{e}^{-a t \varepsilon^{2}}\right] \\
& \quad \leq c_{4}\left(|s|^{2}+\frac{1}{t^{2}}\right)
\end{aligned}
$$

The lemma follows.

The remaining entries in (10.1) can be calculated from (10.3). Setting $\psi_{1}=\psi, \psi_{2}=i \psi$ and $\eta_{1}=\eta$, the substitution $b \mapsto i b$ gives

$$
\left(\eta_{1}, L_{t} \psi_{2}\right)_{C_{\zeta, s}}=-a \operatorname{Re}(i b s \zeta) \mathrm{e}^{-a t|s|^{2} / 4 \varepsilon^{2}}+O\left(\frac{1}{\sqrt{t}}\right)
$$

The entries in the second column of (10.1) are evaluated using similar coordinates $\left(x_{2}, y_{2}, \nu_{2}\right)$ around $q$; in these coordinates $R\left(\nu_{2}\right)=a_{2} \overline{x_{2}} d \overline{x_{2}} \otimes \nu_{2}$ for some real number $a_{2}>0$, and $\psi_{1}$ and $\eta_{2}$ have the form (10.2) with $b$ replaced by a different constant, which we write as $i b_{2} \in \mathbb{C}^{*}$. After a little algebra, one obtains

$\operatorname{det} L_{t, j}=-a a_{2}\left|\begin{array}{cc}\operatorname{Re}\left(i b s \zeta_{j}^{1}\right) & \operatorname{Re}\left(b_{2} s \zeta_{j}^{2}\right) \\ \operatorname{Re}\left(b s \zeta_{j}^{1}\right) & \operatorname{Re}\left(i b_{2} s \zeta_{j}^{2}\right)\end{array}\right|=a a_{2}|s|^{2} \cdot \operatorname{Re}\left(b \overline{b_{2}} \zeta_{j}^{1} \overline{\zeta_{j}^{2}}\right)+O\left(\frac{1}{\sqrt{t}}\right)$.

Proof of Theorem 10.1. By the remark at the end of Section 8 we may assume that $\operatorname{Re}\left(b \bar{b}_{2} \zeta_{j}^{1} \overline{\zeta_{j}^{2}}\right)$ is non-zero for each $j$ with $m_{j}$ even. For these $j$, the above formula gives $\operatorname{sgn} \operatorname{det} L_{t, j}=\operatorname{sgn} \operatorname{Re}\left(b \overline{b_{2}} \zeta_{j}^{1} \overline{\zeta_{j}^{2}}\right)$ when $t$ is large and $0<|s| \leq 1 / t$. For each $\zeta \in Q_{m}$, Theorem 8.2 therefore shows that

$$
p\left(f_{\zeta, s}\right)=(-1)^{h^{0}\left(N_{1}\right)+h^{0}\left(N_{2}\right)} \cdot \prod \operatorname{sgn} \operatorname{Re}\left(b \overline{b_{2}} \zeta_{j}^{1} \overline{\zeta_{j}^{2}}\right)
$$

where the product is over all $j$ with $m_{j}$ even.

Now comes the punch line. Fix an index $j$ with even $m_{j}$. For each $\zeta=\left(\zeta_{1}^{1}, \zeta_{1}^{2}, \ldots, \zeta_{\ell}^{1}, \zeta_{\ell}^{2}\right)$ in $Q_{m}$, replacing $\zeta_{j}^{1}$ by $-\zeta_{j}^{1}$ defines an involution $\iota: Q_{m} \rightarrow Q_{m}$ that reverses the sign of (10.4). Thus the sum

$$
\sum_{\zeta \in Q_{m}} p\left(f_{\zeta, s}\right)=\frac{1}{2} \sum_{\zeta \in Q_{m}}\left[p\left(f_{\zeta, s}\right)+p\left(f_{\iota(\zeta), s}\right)\right]=0
$$


Theorem 10.1 completes the proof of Theorem 1.1 - the main result stated in the introduction. Specifically, Lemmas 5.2 and 8.1 imply Theorems $4.2 \mathrm{a}, 10.1$ and (5.3) imply Theorem $4.2 \mathrm{~b}$, and the arguments at the end of Section 4 showed how Theorem 1.1 follows from Theorem 4.2.

\section{Calculational examples}

This last section uses Theorem 1.1 to explicitly compute the degree $d=4$ spin Hurwitz numbers for every genus. For degrees 1 and 2 the computation is trivial: since the only odd partitions of 1 and 2 are (1) and $\left(1^{2}\right)$, by (4.4) the degree $d=1,2$ spin Hurwitz numbers are the etale spin Hurwitz numbers

$$
H_{1}^{h, p}=(-1)^{p}, \quad H_{2}^{h, p}=(-1)^{p} 2^{h}
$$

which are the GW invariants of Kähler surfaces calculated in [10] and [8]. For notational simplicity, we will write the spin Hurwitz numbers $H_{m, \ldots, m}^{h, p}$ with the same $k$ partitions $m$ of $d$ simply as $H_{m^{k}}^{h, p}$ and the etale spin Hurwitz number $H_{d}^{h, p}$ as $H_{m^{0}}^{h, p}$. The numbers 3 and 4 each have two odd partitions, namely (3) and $\left(1^{3}\right)$, and $(31)$ and $\left(1^{4}\right)$. Thus, by (4.4), it suffices to compute $H_{(3)^{k}}^{h, p}$ and $H_{(31)^{k}}^{h, p}$ for all $k \geq 0$. The degree $d=3$ case is calculated in [9]:

$$
H_{(3)^{k}}^{h, \pm}=3^{2 h-2}\left[(-1)^{k} 2^{k+h-1} \pm 1\right]
$$

where + and - denote the even and odd parities. Here, we will compute the corresponding degree 4 invariants.

Theorem 11.1. The degree 4 Hurwitz numbers are

$$
H_{(31)^{k}}^{h, \pm}=(3 !)^{2 h-2} \cdot 2^{k}\left[ \pm 2^{k+h-1}+(-1)^{k}\right] \quad \text { for } k \geq 0
$$

We begin by computing three special cases.

Lemma 11.2. (a) $H_{4}^{1,-}=0$, (b) $H_{(31)}^{1,-}=-6$ and (c) $H_{(31)^{3}}^{0,+}=\frac{2}{3}$.

Proof. For a genus one spin curve with odd parity, formula (3.12) of [3] shows that

$$
H_{(31)^{k}}^{1,-}=2^{-k}\left[\left(\mathbf{f}_{(3)}(31)\right)^{k}-\left(\mathbf{f}_{(3)}(4)\right)^{k}\right]
$$


Here the so-called central character $\mathbf{f}_{(3)}$ can be written as $\mathbf{f}_{(3)}=\frac{1}{3} \mathbf{p}_{3}+$ $a_{2} \mathbf{p}_{1}^{2}+a_{1} \mathbf{p}_{1}+a_{0}$ for some $a_{i} \in \mathbb{Q}$ and $\mathbf{p}_{1}$ and $\mathbf{p}_{3}$ are the functions of partitions $m=\left(m_{1}, \ldots, m_{\ell}\right)$ of $d$ defined by

$$
\mathbf{p}_{1}(m)=d-\frac{1}{24} \quad \text { and } \quad \mathbf{p}_{3}(m)=\sum_{j} m_{j}^{3}-\frac{1}{240}
$$

The case $k=0$ gives (a), and the case $k=1$ gives (b).

Next consider a map $f$ in the dimension zero relative moduli space $\mathcal{M}_{\chi,(31),(31),(31)}^{V}\left(\mathbb{P}^{1}, 4\right)$. By the dimension formula $(4.2), \chi=2$ and hence the domain of $f$ is either a rational curve or a disjoint union of a rational curve $C_{0}$ and an elliptic curve $C_{1}$. Maps of the first type have parity $p(f)=1$ since $N_{f}=\mathcal{O}(-1)$. For maps of the second type,

- $f_{0}=\left.f\right|_{C_{0}} \in \mathcal{M}_{2,(1),(1),(1)}^{V}\left(\mathbb{P}^{1}, 1\right)$ and $N_{f_{0}}=\mathcal{O}(-1)$,

- $f_{1}=\left.f\right|_{C_{1}} \in \mathcal{M}_{0,(3),(3),(3)}^{V}\left(\mathbb{P}^{1}, 3\right)$ and $N_{f_{1}}=\mathcal{O}$ (cf. the proof of Lemma $7.2 \mathrm{~b}$ of $[9])$.

It follows that $p(f)=p\left(f_{0}\right) \cdot p\left(f_{1}\right)=1 \cdot(-1)=-1$. Thus by (1.2) and (1.7) the difference between the ordinary and spin Hurwitz numbers is twice the contribution of the maps of the second type:

$$
H_{(31)^{3}}^{0,+}=H_{(31)^{3}}^{0}-2 H_{(1)^{3}}^{0} \cdot H_{(3)^{3}}^{0} \cdot
$$

The three (ordinary) Hurwitz numbers on the right-hand side can be calculated by using formula (0.10) of [12]. This yields (c).

Lemma 11.3. Theorem 11.1 holds for genus $h=0$ and genus $h=1$.

Proof. Taking $h=h_{1}=1$ and $p=p_{1}=1$ in Theorem 1.1a and using Lemma 11.2 gives

$$
H_{(31)^{2}}^{1,-}=3 H_{(31)}^{1,-} \cdot H_{(31)^{3}}^{0,+}=-12
$$

Using (11.2) and Lemma $11.2 \mathrm{~b}$ to evaluate the $k=1$ and $k=2$ cases of (11.1), one sees that $\mathbf{f}_{(3)}(31)=-4$ and $\mathbf{f}_{(3)}(4)=8$. Formula (11.1) then becomes

$$
H_{(31)^{k}}^{1,-}=(-1)^{k} 2^{k}-4^{k} \quad \text { for } k \geq 0
$$


For $k \geq 1$, we can apply Theorem 1.1a with $\left(h_{1}, p_{1}\right)=(1,-),\left(h_{2}, p_{2}\right)=$ $(0,+)$ and $k_{0}=0$ and use Lemma 11.2 a to obtain

$$
H_{(31)^{k-1}}^{1,-}=3 H_{(31)}^{1,-} \cdot H_{(31)^{k}}^{0,+}=-3 \cdot 3 ! H_{(31)^{k}}^{0,+} .
$$

Together with (11.3), this equation yields

$$
H_{(31)^{k}}^{0,+}=-\frac{1}{3 \cdot 3 !}\left[(-1)^{k-1} 2^{k-1}-4^{k-1}\right] \text { for } k \geq 1
$$

and the same formula holds for $k=0$ because the invariant $H_{(31)^{0}}^{0,+}=H_{4}^{0,+}$ is $\frac{1}{4 !}$. Finally, combining (11.4) with the formula of Theorem $1.1 \mathrm{~b}$ with $(h, p)=$ $(1,+)$, shows that

$$
H_{(31)^{k}}^{1,+}=3 H_{(31)^{k+2}}^{0,+}+4 ! H_{(31)^{k}}^{0,+}=(-1)^{k} 2^{k}+4^{k} .
$$

Proof of Theorem 11.1: By Lemma 11.3 we can assume that $h \geq 2$. Applying the formula of Theorem 1.1a with $\left(h_{2}, p_{2}\right)=(1,+)$, we obtain

$$
H_{(31)^{k}}^{h, p}=4 ! H_{(31)^{0}}^{h-1, p} \cdot H_{(31)^{k}}^{1,+}+3 H_{(31)}^{h-1, p} \cdot H_{(31)^{k+1}}^{1,+} .
$$

From this, we can deduce the matrix equation

$$
\left(\begin{array}{c}
H_{(31)^{k}}^{h, p} \\
H_{(31)^{k+1}}^{h, p}
\end{array}\right)=\left(\begin{array}{cc}
4 ! H_{(31)^{k}}^{1,+} & 3 H_{(31)^{k+1}}^{1,+} \\
4 ! H_{(31)^{k+1}}^{1,+} & 3 H_{(31)^{k+2}}^{1,+}
\end{array}\right)\left(\begin{array}{cc}
4 ! H_{(31)^{0}}^{1,+} & 3 H_{(31)}^{1,+} \\
4 ! H_{(31)}^{1,+} & 3 H_{(31)^{2}}^{1,+}
\end{array}\right)^{h-2}\left(\begin{array}{c}
H_{(31)^{0}}^{1, p} \\
H_{(31)}^{1, p}
\end{array}\right) .
$$

Theorem 11.1 follows after inserting the values given by (11.3) and (11.5).

\section{Acknowledgment}

This work was partially supported by N.S.F. grants DMS-1206192 (J.L.) and DMS-1011793 (T.P.).

\section{Appendix A.}

This appendix establishes the subjectivity statement needed in the proof of Theorem 7.2. Let $\mathcal{E}$ (resp. $E_{W}$ ) be the image of the map $\Phi_{s}\left(\operatorname{resp} . \Phi_{s}^{W}\right.$ ) defined below (7.13). 
Lemma A.1. Given $0<T$, there are constants $c_{0}, \delta>0$ such that whenever $|s|$ is sufficiently small all eigenspaces $E_{\lambda}$ with $\lambda|\log | s||<c_{0}$ satisfy

$$
\text { (a) } E_{\lambda} \subset \mathcal{E} \text { for }|t| \leq \delta \quad \text { (b) } E_{\lambda} \subset \mathcal{E}_{W} \text { for } T<|t|
$$

Proof. Otherwise there would be sequences $t_{n} \rightarrow \tau$ and $s_{n} \rightarrow 0$ and $L^{2}$ normalized eigensections $\xi_{n}$ on $C_{n}=C_{s_{n}}$ with eigenvalues satisfying $\lambda_{n} \mid \log$ $\left|s_{n}\right| \mid \rightarrow 0$ and with $L^{2}$ orthogonal to $\mathcal{E}$ on $C_{n}$ with $t_{0}=0$ in case (a), and $L^{2}$ orthogonal to $\mathcal{E}_{W}$ with $\tau \geq T$ in case (b). By (2.5) the $L^{2}$ norms satisfy

$$
\left\|\bar{\partial} \xi_{n}\right\|^{2}+t^{2}\left\|R \xi_{n}\right\|^{2}=\left\|L_{t_{n}} \xi_{n}\right\|^{2}=\lambda_{n} \rightarrow 0
$$

as $n \rightarrow \infty$. On any compact set $K \subset \mathcal{C} \backslash\left\{\right.$ nodes of $\left.C_{0}\right\}$ we can use the coordinates of Section 5 to identify $K \cap C_{s}$ with $K \cap C_{0}$ and regard $\xi_{n}$ as a section on $K \cap C_{0}$. Under this identification, the geometry of $K \cap C_{s}$ converges to that of $K \cap C_{0}$. An elliptic estimate for $\bar{\partial}$ then provides a bound on the Sobolev $W^{1,2}$ norm of $\xi_{n}$ :

$$
\int_{C_{n}}\left|\nabla \xi_{n}\right|^{2}+\left|\xi_{n}\right|^{2} \leq c_{1} \int_{C_{n}}\left|\bar{\partial} \xi_{n}\right|^{2}+\left|\xi_{n}\right|^{2} \leq c_{2}\left(\lambda_{n}+1\right) \leq 2 c_{2}
$$

for large $n$. Therefore, by elliptic theory, a subsequence converges in $L^{2}(K)$ and weakly in $W^{1,2}(K)$ to a limit $\xi_{0}$ with $L_{\tau}^{*} L_{\tau} \xi_{0}=0$. Applying this argument for a sequence of compact sets $K$ that exhaust $\mathcal{C} \backslash\{$ nodes $\}$ and repeatedly extracting subsequences yields a solution of $L_{\tau} \xi_{0}=0$ on $C_{0} \backslash\{$ nodes . By a standard argument (see the proof of Lemma 7.6 in [11]) $\xi_{0}$ extends over the nodes in the normalization of $C_{0}$ to a solution of $L_{\tau} \xi_{0}=0$. Theorem 2.2 then implies that $\xi_{0}$ is holomorphic.

To show $\xi_{0}$ is non-trivial we must rule out the possibility that the $L^{2}$ norm of $\xi_{n}$ accumulates at the nodes. Fix a node $p$ of $C_{0}$, a local holomorphic section $\nu$ of $\mathcal{N}$ with $\frac{1}{2} \leq|\nu|^{2} \leq 2$ pointwise on $C_{n}(2 \varepsilon)=B(p, 2 \varepsilon) \cap C_{n}$, and coordinates $(x, y)$ around $p$ in which $C_{n}=\left\{x y=\zeta s_{n}\right\}$. Then the functions $f_{n}$ defined by $\xi_{n}=f_{n} \nu$ satisfy $\left|\xi_{n}\right|^{2} \leq 2\left|f_{n}\right|^{2}$ and $\left|\bar{\partial} f_{n}\right|^{2} \leq 2\left|\bar{\partial} \xi_{n}\right|^{2}$ on $C_{n}$. Lemma A.2 below and (A.2) show that

$$
\int_{C_{n}(\varepsilon)}\left|\xi_{n}\right|^{2} \leq c_{4} \varepsilon^{2} \int_{C_{n}}\left|\bar{\partial} \xi_{n}\right|^{2}+c_{5} \int_{C_{n}(2 \varepsilon) \backslash C_{n}(\varepsilon)}\left|\xi_{n}\right|^{2} \leq c_{4} \varepsilon^{2} \lambda_{n}+c_{5} \int_{K}\left|\xi_{n}\right|^{2}
$$

with $\lambda_{n} \rightarrow 0$. If $\xi_{0}=0$ then the last integral also vanishes as $n \rightarrow \infty$ because $\xi_{n} \rightarrow \xi_{0}=0$ in $L^{2}(K)$. Thus, the $L^{2}$ norm does not accumulate at any node, which implies that $\left\|\xi_{0}\right\|=\lim _{n \rightarrow \infty}\left\|\xi_{n}\right\|=1$; this is a contradiction unless $\xi_{0} \neq 0$. 
Furthermore, $\xi_{0}$ is continuous, as follows. Fix a node $p$, a local holomorphic trivialization of $\mathcal{N} \rightarrow \mathcal{C}$ around $p$, and local coordinates in which $C_{s}$ is given by $x y=\zeta s$ and regard $\xi_{0}$ as a holomorphic function. Let $p^{\prime}$ and $p^{\prime \prime}$ be the points in the normalization above $p$ and let $A_{n}$ be the annular region on $C_{n}$ between the circles $\gamma_{1}(s)=\{x=1\}$ and $\gamma_{2}(s)=\{y=1\}$. Setting $\eta=x^{-1} d x=-y^{-1} d y$ we have

$$
2 \pi \mathrm{i} \xi_{0}\left(p^{\prime}\right)=\int_{\gamma_{1}(0)} \xi \eta=\lim _{n \rightarrow \infty} \int_{\gamma_{1}\left(s_{n}\right)} \xi_{n} \eta .
$$

and similarly for $\xi_{0}\left(p^{\prime \prime}\right)$. Setting $r=|x|$ and noting that $|\eta|_{g}^{2} d v_{g}$ is conformally invariant (cf. Lemma A.2), we have

$$
\begin{aligned}
2 \pi\left|\xi_{0}\left(p^{\prime}\right)-\xi_{0}\left(p^{\prime \prime}\right)\right| & \leq \varlimsup \\
& \leq \varlimsup \lim \left(2 \pi \lambda_{n}|\log | s_{n}||\right)^{\frac{1}{2}}=0
\end{aligned}
$$

Thus $\xi_{0}$ is a continuous element of ker $L_{\tau}$ on $C_{0}$. Lemma 7.1 then implies that $\xi_{0} \in \mathcal{E}_{0}$ in case (a) and $\xi_{0} \in W$ in case (b).

But in case (a) each $\xi_{n}$ is $L^{2}$ orthogonal to $\mathcal{E}_{s_{n}}$ on $C_{n}$. For the basis $\left\{\psi_{k, s}\right\}$ in (7.10), one sees that for each $\delta>0$ there is a compact set $K$ so that the $L^{2}$ norm of $\psi_{k, s}$ on $C_{n} \backslash K$ is less than $\delta$, uniformly in $s$. A simple estimate then shows that $\xi_{0}$ is $L^{2}$ orthogonal to $\mathcal{E}_{0}$. Likewise, in case (b) one sees that $\xi_{0}$ is $L^{2}$ orthogonal to $W$. This contradicts our previous conclusion about $\xi_{0}$, completing the proof.

Lemma A.2. Let $C_{s}(2 \varepsilon)$ be the curve $\{x y=\zeta s|| x|<2 \varepsilon| y \mid,<2 \varepsilon\}$ in $\mathbb{C}^{2}$ with the induced Riemannian metric. Then there are constants $c_{1}$ and $c_{2}$, independent of $s$ and $\varepsilon$, such that every smooth function $f$ on $C_{s}$ satisfies

$$
\int_{C_{s}(\varepsilon)}|f|^{2} \leq c_{1} \varepsilon^{2} \int_{C_{s}(2 \varepsilon)}|\bar{\partial} f|^{2}+c_{2} \int_{C_{s}(2 \varepsilon) \backslash C_{s}(\varepsilon)}|f|^{2} .
$$

Proof. A simple calculation shows that the Riemannian metric $g_{s}$ on $C_{s}$ is conformal to the euclidean metric in the $x$-coordinate:

$$
g_{s}=\gamma^{2} d x^{2} \quad \text { where } \gamma^{2}=1+\frac{s^{2}}{r^{4}}, \quad r=|x|
$$

Fix a smooth cutoff function $\beta(\rho), \quad \rho^{2}=|x|^{2}+|y|^{2}$, supported on $B=B(2 \varepsilon) \subset \mathbb{C}^{2}$ with $\beta=1$ on $B(\varepsilon), 0 \leq \beta \leq 1$ and $|d \beta| \leq 2 / \varepsilon$ pointwise. 
Then $h=\beta f$ is a smooth function of $x$ that vanishes on $\partial B$. Setting $\phi=$ $\frac{1}{2}\left(r^{2}-s^{2} / r^{2}\right)$, we have $d \operatorname{vol}_{s}=\phi^{\prime} d r d \theta$ by (A.3) and can integrate by parts:

$$
I=\int_{B}|h|^{2} d \operatorname{vol}_{s}=\int_{B}|h|^{2} \phi^{\prime} d r d \theta \leq \int_{B}|h||d h| 2 \phi d r d \theta .
$$

But $2 \phi \leq r^{2} \gamma^{2}=\rho^{2}$ with $\rho \leq 2 \varepsilon$ so, continuing using Cauchy-Schwarz and $d \operatorname{vol}_{s}=\gamma^{2} r d r d \theta$,

$$
I \leq \int_{B}|h| \gamma \rho \sqrt{r} \cdot|d h| \sqrt{r} d r d \theta \leq 2 \varepsilon \sqrt{I}\left(\int_{B}|d h|^{2} r d r d \theta\right)^{1 / 2}
$$

The last integrand is conformally invariant, so can be replaced by $|d h|_{g}^{2} d v_{g}$. Rearranging, we have $I \leq 4 \varepsilon^{2}\|d h\|^{2} \leq 8 \varepsilon^{2}\|\bar{\partial} h\|^{2}$ where this second inequality is obtained by integrating by parts using the formula $2 \bar{\partial}^{*} \bar{\partial}=d^{*} d$. The lemma follows because $|\bar{\partial} h|^{2} \leq 2\left(|\bar{\partial} \beta|^{2}|f|^{2}+|\bar{\partial} f|^{2}\right)$ where $d \beta$ has support on $C_{s}(2 \varepsilon) \backslash C_{s}(\varepsilon)$.

\section{References}

[1] E. Arbarello, M. Cornalba, P. Griffiths and J. Harris, Geometry of Algebraic Curves: Volume II, Springer-Verlag, Berlin, 2011.

[2] M. Cornalba, Moduli of curves and theta characteristics, Lectures on Riemann Surfaces, 560-589, World Scientific, Singapore 1989.

[3] A. Eskin, A. Okounkov and R. Pandharipande, The theta characteristic of a branched covering, Adv. Math. 217(3) (2008), 873-888.

[4] S. Gunningham, Spin Hurwitz numbers and topological quantum field theory, arXiv:1201.1273.

[5] J. Harris and I. Morrison, Moduli of Curves, Springer, New York, 1998.

[6] E. Ionel and T.H. Parker, Relative Gromov-Witten Invariants, Ann. Math. 157 (2003), 45-96.

[7] E. Ionel and T.H. Parker, The symplectic sum formula for GromovWitten invariants, Ann. Math. 159 (2004), 935-1025.

[8] Y-H. Kiem and J. Li, Low degree $G W$ invariants of spin surfaces, Pure Appl. Math. Q. 7(4) (2011), 1449-1476.

[9] J. Lee, Degree three spin Hurwitz numbers, Pacific J. Math. 263(2) (2013), 399-417. 
[10] J. Lee and T.H. Parker, A structure theorem for the Gromov-Witten invariants of Kähler surfaces, J. Differ. Geom. 77(3) (2007), 483-513.

[11] J. Lee and T.H. Parker, An obstruction bundle relating Gromov-Witten invariants of curves and Kähler surfaces, Amer. J. Math. 134(2) (2012), $453-506$.

[12] A. Okounkov, R. Pandharipande, Gromov-Witten theory, Hurwitz theory, and completed cycles, Ann. Math. (2) 163(2) (2006), 517-560.

Department of Mathematics

University of Central Florida

ORLANDO

FL 32816

USA

E-mail address: Junho.Lee@ucf .edu

Department of Mathematics

Michigan State University

EAST LANSING

MI 48824

USA

E-mail address: parker@math.msu.edu

ReCEIVEd January 29, 2013 